\title{
Molecular and genetic organization of bands and interbands in the dot chromosome of Drosophila melanogaster
}

\author{
Darya S. Sidorenko ${ }^{1} \cdot$ Ivan A. Sidorenko ${ }^{2} \cdot$ Tatyana Yu. Zykova $^{1} \cdot$ Fedor P. Goncharov ${ }^{1} \cdot$ Jan Larsson ${ }^{3}$. \\ Igor F. Zhimulev ${ }^{1,4}$
}

Received: 9 August 2018 / Revised: 9 January 2019 / Accepted: 2 April 2019 / Published online: 30 April 2019

(C) The Author(s) 2019

\begin{abstract}
The fourth chromosome smallest in the genome of Drosophila melanogaster differs from other chromosomes in many ways. It has high repeat density in conditions of a large number of active genes. Gray bands represent a significant part of this polytene chromosome. Specific proteins including HP1a, POF, and dSETDB1 establish the epigenetic state of this unique chromatin domain. In order to compare maps of localization of genes, bands, and chromatin types of the fourth chromosome, we performed FISH analysis of 38 probes chosen according to the model of four chromatin types. It allowed clarifying the dot chromosome cytological map consisting of 16 loose gray bands, 11 dense black bands, and 26 interbands. We described the relation between chromatin states and bands. Open aquamarine chromatin mostly corresponds to interbands and it contains 5 'UTRs of housekeeping genes. Their coding parts are embedded in gray bands substantially composed of lazurite chromatin of intermediate compaction. Polygenic black bands contain most of dense ruby chromatin, and also some malachite and lazurite. Having an accurate map of the fourth chromosome bands and its correspondence to physical map, we found that DNase I hypersensitivity sites, ORC2 protein, and $P$-elements are mainly located in open aquamarine chromatin, while element 1360 , characteristic of the fourth chromosome, occupies band chromatin types. POF and HP1a proteins providing special organization of this chromosome are mostly located in aquamarine and lazurite chromatin. In general, band organization of the fourth chromosome shares the features of the whole Drosophila genome.
\end{abstract}

Keywords Drosophila $\cdot$ Polytene chromosomes $\cdot$ Dot chromosome $\cdot$ Chromatin types $\cdot$ Bands $\cdot$ Interbands

\section{Introduction}

Genetic activity of interphase chromosomes depends on their structural organization, but the basis of this interconnection has not been studied in sufficient detail.

Electronic supplementary material The online version of this article (https://doi.org/10.1007/s00412-019-00703-x) contains supplementary material, which is available to authorized users.

Igor F. Zhimulev

zhimulev@mcb.nsc.ru

1 Institute of Molecular and Cellular Biology of the Siberian Branch of the Russian Academy of Sciences, Lavrentiev Ave. 8/2, Novosibirsk, Russia 630090

2 Institute of Cytology and Genetics of the Siberian Branch of the Russian Academy of Sciences, Novosibirsk, Russia

3 Department of Molecular Biology, Umeå University, Umeå, Sweden

4 Laboratory of structural, functional and comparative genomics of the Novosibirsk State University, Novosibirsk, Russia
Polytene chromosomes of Drosophila melanogaster are a convenient model for such studies. In contrast to the chromosomes of diploid cells, polytene chromosomes can be observed along their entire length in the interphase nucleus. These structures are formed due to multiple rounds of DNA replication without subsequent segregation of daughter chromatids. For this reason, the chromomeric pattern becomes represented in the form of alternating transverse stripesblack compact bands, less compact gray bands, and loose light-colored interbands. Due to the unique polytene chromosome banding pattern, C. Bridges performed detailed chromosome drawings with simple symbols (Bridges 1935a). These maps are actively used in research at the present time.

Despite obvious morphological features, polytene chromosomes demonstrate universal principle of interphase chromosome organization in all cell types, which has been shown in detail in our previous works (Zhimulev et al. 2014; Zykova et al. 2018). Giant size of polytene chromosomes and the banding pattern, unique for each region, allow connecting molecular and genetic characteristics of chromatin with 
morphological organization of interphase chromosomes with high resolution. To achieve this goal, it is necessary to localize the DNA sequence on the cytological map of polytene chromosomes.

Sequencing of the D. melanogaster genome has provided an opportunity to study structural and functional organization of chromosomes in more detail. So far, a large amount of genomic data on localization of proteins and regulatory elements on interphase chromosomes in cell cultures undergoing mitosis have been accumulated in the modENCODE project (model organism Encyclopedia of DNA Elements). Recently, researchers have faced the problem of transforming the obtained information into functional maps that characterize the processes of transcription, replication, splicing, and epigenetic changes regulation (The modENCODE Consortium et al. 2010). In recent studies, various types of Drosophila chromatin have been distinguished using computer modeling and the methods of DamID (DNA adenine methyltransferase identification), CHIP-on-chip (chromatin immunoprecipitation on chip), and the determination of the overall chromatin sensitivity to DNase I (Filion et al. 2010; Kharchenko et al. 2011; Milon et al. 2014). The DamID definition of five chromatin types is based on the distribution of 53 diverse proteins obtained in DamID experiments (Filion et al. 2010). The authors note that the fourth chromosome (and pericentric heterochromatin) is mainly enriched in GREEN chromatin that corresponds to classic heterochromatin marked by SU(VAR)3-9, HP1, and the HP1-interacting proteins LHR and HP6 (Filion et al. 2010). The modENCODE consortium identified the prevalent combinatorial patterns of 18 histone modifications (CHIP-on-chip data) and determined nine combinatorial chromatin states (Kharchenko et al. 2011). The fourth chromosome and pericentric heterochromatin are characterized by high levels of H3K9me2/me3 (state 7 , dark blue) (Kharchenko et al. 2011). The 3CM model created by Milon et al. (2014) is based on general chromatin sensitivity to DNase I. This model revealed three states: open, closed, and neutral (Milon et al. 2014). According to 3CM, chromosome 4 and pericentric heterochromatin are enriched in neutral chromatin. Therefore, chromosome 4 appears to share similarities with both euchromatin and heterochromatin (Milon et al. 2014). However, the correlation of these chromatin types (Filion et al. 2010; Kharchenko et al. 2011; Milon et al. 2014) based on different input data with morphological structures of polytene chromosomes remains a topic of current interest.

In our laboratory, we developed a unique approach of determining interband localization in the genome of Drosophila. It is based on $P$-element-mediated transformation of DNA, which results in compaction of the construction inserted. If the insertion occurs in a band, there are no changes in polytene chromosome structure. In case of insertion localization in an interband, a new band is formed (Semeshin et al. 1986;
Demakov et al. 1993; Vatolina et al. 2011b). Sequencing the DNA surrounding the insertions allowed us to determine the genomic position of 12 interbands. Using modENCODE data on the distribution of chromatin proteins in mitotically dividing cells, we defined a set of proteins that enrich DNA sequences corresponding to these interbands previously located on the physical map of the Drosophila genome (Demakov et al. 2011; Vatolina et al. 2011a). Next, an algorithm based on the distribution of these open chromatin proteins that allows determining the interband localization in the large arms of D. melanogaster polytene chromosomes was developed. Using this algorithm, four types of chromatin, conventionally named cyan, blue, magenta, and green, were identified (Zhimulev et al. 2014). Later, in order to avoid confusing caused by the intersection of names with other works, these chromatin types were renamed aquamarine, lazurite, ruby, and malachite, respectively (Khoroshko et al. 2016). The advantage of this model is a good correspondence of these chromatin types to a number of bands and interbands with already determined position on the molecular map. Aquamarine chromatin occupies about $13 \%$ of the genome and is enriched with open chromatin proteins. This type of chromatin represents interbands previously localized on the physical map of the genome. Ruby chromatin occupies almost half of the genome and is completely devoid of open chromatin proteins. It corresponds to black bands and intercalary heterochromatin bands. Malachite and lazurite do not demonstrate specificity about the "model" open chromatin proteins; they often correspond to gray bands (for more details about the model, see (Zhimulev et al. 2014; Boldyreva et al. 2017; Zykova et al. 2018)).

Thus, the model of four chromatin states allows determining the localization of interbands and bands on the physical map of the D. melanogaster genome, a problem that has not been solved using other chromatin state models. After matching cytological and physical maps, it is possible to investigate the molecular characteristics of these polytene chromosome morphological structures using whole genome data. In the present work, we focused on chromatin organization of the D. melanogaster fourth chromosome.

The fourth chromosome is the smallest one in the Drosophila genome. Most of the $4.2 \mathrm{Mb}$ fourth chromosome is dense heterochromatin; the euchromatic arm of this chromosome occupies only 1.2 Mb (Locke and McDermid 1993). Therefore, this chromosome looks like a "dot" on the metaphase chromosome preparations (Gatti et al. 1976; Pimpinelli et al. 1976; Pokholkova et al. 1993). After numerous cycles of endoreplication, in the polyploid nuclei of salivary gland cells, large blocks of heterochromatin become underrepresented, and the small euchromatic arm of the dot chromosome becomes visible. C. Bridges divided the small fourth chromosome into 101 and 102 sections and letter subsections; however, the cytological map has not been completed, and the 
bands did not receive individual numbers. A large number of gray bands that have an intermediate degree of compaction and the tendency of its tip to conjugate with the chromocenter ectopically causes a certain complexity in the mapping of the polytene fourth chromosome. In the current investigation, for a better spreading of the polytene dot chromosome on the preparations, we used the fly stock with suppressed underreplication.

The fourth chromosome of Drosophila has an unusual organization. This chromosome shares the characteristics of both eu- and heterochromatin and a number of unique properties (Riddle and Elgin 2006; Riddle et al. 2009). Gene density in this chromosome corresponds to euchromatin. At the same time, there is no recombination in the dot chromosome (Sandler and Szauter 1978). The absence of crossing-over leads to accumulation of repeated sequences and mobile elements of the genome. Repeat density in the fourth chromosome is similar to that in pericentric heterochromatin (Slawson et al. 2006). The 1360 (Kholodilov et al. 1988) and DINE-1 (Locke et al. 1999) elements are predominantly concentrated on this chromosome. The transgenic constructs based on $P$ transposons often demonstrate variegating phenotype of the reporter gene (Sun et al. 2000; Riddle et al. 2008; for more details about position effect variegation, see Elgin and Reuter 2013). In addition, such heterochromatin mark as methylation of H3K9, which is mainly introduced by specific for this chromosome histone methyltransferase dSETDB1 (Tzeng et al. 2007; Figueiredo et al. 2012), is associated with the fourth chromosome. Typical heterochromatin protein HP1a that recognizes H3K9 methylation binds the fourth chromosome (James et al. 1989), although its role in chromatin organization of this chromosome is still subject to discussion.

A unique feature of the fourth chromosome is POF (painting-of-fourth) protein presumably evolutionarily associated with the dose compensation system that $D$. melanogaster adapted to regulate the work of the fourth chromosome genes in heterochromatic environment (Larsson et al. 2001). POF can enhance transcription or RNA transport through nuclear pores by binding to nascent RNA (Johansson et al. 2012). Thus, an interactive network of epigenetic chromatin regulation, unique for this autosome, including dSETDB1, HP1a, and POF, is involved in maintaining the activity of the fourth chromosome genes in the heterochromatic environment (Seum et al. 2007; Tzeng et al. 2007; Brower-Toland et al. 2009) (for more details about the fourth chromosome organization, see Riddle and Elgin (2018)). We were interested to know whether the band organization of the fourth chromosome is influenced by the combination of characteristics mentioned above that distinguish the chromosome under investigation from other Drosophila autosomes.

In our work, we applied a combined approach of mapping the entire sections of polytene chromosomes using computer modeling, modENCODE data, electron microscopy, fluorescent in situ hybridization (FISH), and light microscopy to determine the genomic coordinates of the fourth chromosome bands and interbands, and clarified its cytological map. This allowed us to study in detail the genetic organization, the distribution of chromatin proteins, mobile genetic elements, and other elements of the genome in the morphological structures of this unique $D$. melanogaster chromatin domain using whole genome databases.

\section{Materials and methods}

\section{Map of chromatin types}

Four basic chromatin types (aquamarine, lazurite, malachite, and ruby) used in the current study were defined earlier in five large chromosome arms 2L, 2R, 3L, 3R, and X (Zhimulev et al. 2014; Boldyreva et al. 2017; Zykova et al. 2018). Aquamarine chromatin mostly matches interbands, ruby chromatin vice versa corresponds to dense black bands, and lazurite and malachite chromatin have an intermediate level of compactness and coincide with gray bands (Zhimulev et al. 2014; Zhimulev et al. 2016; Boldyreva et al. 2017). In this study, the same four chromatin types were identified in the fourth chromosome. Each chromatin type was composed of 200-bp-long non-overlapping fragments belonging to the euchromatic portion of the fourth chromosome. There are also some regions named gaps where the model failed to return a specific value. The model was smoothed: we combined the intervals of identical states if gaps between them did not exceed $400 \mathrm{bp}$. Using this four-chromatin-state model, the chromatin composition of the fourth chromosome morphological structures was determined.

\section{Fly stock}

In the present work we used a fly stock with $\hat{X Y X Y} / \hat{X Y} ; y$ w; $S u U R^{E S}$, Su(var)3-9 $9^{06}$ genotype because of suppressed underreplication of chromosome regions for better spreading of Drosophila fourth polytene chromosome on preparations (Andreyeva et al. 2007; Demakova et al. 2007). Flies were raised on standard cornmeal-yeast-agar-molasses medium (Semeshin et al. 2004). The fly stock was kindly provided by E. S. Belyaeva.

\section{Fluorescent in situ hybridization}

Salivary glands were dissected in PBS solution and then fixed in a $3: 1$ mixture of ethanol and acetic acid for $30 \mathrm{~min}$ at $20{ }^{\circ} \mathrm{C}$, squashed in $45 \%$ acetic acid, snap-frozen in liquid nitrogen, and stored in $70 \%$ ethanol at $-20^{\circ} \mathrm{C}$. Squashed polytene chromosome preparations together with electron 
microscopy images were used to refine the fourth chromosome cytological map. FISH on polytene chromosomes was performed as described (Ashburner et al. 2005). Thirty-eight DNA probes were selected according to the four chromatin states and obtained by standard PCR. Random-primed labeling of DNA probes with TAMRA or Fluorescein (Biosan) was done using Klenow enzyme. All the probes used in this study are described in Table S1 (probes from bands predicted by the four-chromatin-state model) and in Table S2 (probes from open chromatin predicted by the four-chromatin-state model). FISH preparations were analyzed using fluorescent microscopy and the probes were plotted on the cytological map by C. Bridges.

\section{Immunostaining of polytene chromosomes}

The preparations were made according to Czermin et al. (2002). Immunostaining was performed using anti-POF antibodies (1:100; Larsson et al. 2001), anti-CHRIZ antibodies (1:600; Gortchakov et al. 2005), and fluorescent secondary antibodies. Images were taken with fluorescent microscope.

\section{Genetic organization of the fourth chromosome bands and interbands}

The genetic content of the fourth chromosome morphological structures was analyzed using FlyBase release 5.50. Gene expression was determined according to FlyAtlas as the number of tissues where the particular gene is expressed (Chintapalli et al. 2007).

\section{Analysis of protein distribution in the fourth chromosome}

In our work, we used the ChIP-chip and ChIP-seq data on protein mapping from the "Chromosomal Proteins" and "Regulatory Elements in Drosophila" projects by the modENCODE consortium (release 33).

Protein enrichment in the fourth chromosome chromatin types was compared to that in the whole genome. All analysis was performed in the R statistical environment version 3.3 and Bioconductor packages (Lawrence et al. 2009; Lawrence et al. 2013; R Core Team 2017). All statistical tests were two-sided and statistical significance was determined if $p$ value was less than 0.001. Comparisons were made using the unpaired Wilcoxon test.

The distribution of ORC2 (modENCODE data) in different chromatin types and morphological structures of the fourth chromosome was analyzed in more detail using the algorithm we wrote in $\mathrm{C}++$.

The program algorithm was as follows. First, we sorted all genes, and selected those that fell into the region under investigation. From the start of the $5^{\prime}$ UTRs of these genes, we divided the region under investigation into fragments of $200 \mathrm{bp}$ towards the gene and the intergenic spacer. Next, we counted the number of ORC2 binding sites in each fragment and normalized it to the number of gene transcripts, wherein we took into account different variants of gene localization in the region under investigation, including "head-to-head" orientation.

HP1a and POF distribution in S2 cells (modENCODE data) was studied using the UCSC Table Browser intersection tool (http://bit.ly/TableBrowserTool). Intersection was performed with chromatin types and morphological structures of the fourth chromosome.

POF (Lundberg et al. 2013a; Johansson and Larsson 2014) and H3K27me3 (Sher et al. 2012) distribution in larval salivary glands was studied using Galaxy tools (https://usegalaxy.org/).

SUUR distribution at the cytological level was studied using microscopy images kindly provided by Tatyana D. Kolesnikova.

\section{Analysis of transposable element insertion site distribution in the fourth chromosome}

To analyze the distribution of $P$-element insertions within the euchromatic part of the fourth chromosome, we used insertion coordinates tagged "transposable_element_insertion_site" from FlyBase release 5.50 (107 transposable element insertion sites in the chromosome 4). Its distribution was analyzed in the four chromatin types and morphological structures of the fourth chromosome. The algorithm was the same as in the case of ORC2.

The amount of different chromatin types and morphological structures of the fourth chromosome occupied by the element 1360 and its remnants (59 items, modENCODE data) was determined using the UCSC Table Browser intersection tool (http://bit.ly/TableBrowserTool).

\section{Analysis of DNasel hypersensitive sites distribution in the fourth chromosome}

The distribution of DNaseI hypersensitivity sites (DHS) was analyzed in more detail using the algorithm we wrote in $\mathrm{C}++$. The algorithm was the same as in the case of ORC2.

Data availability The datasets generated during and/or analyzed during the current study are available from the corresponding author on reasonable request.

\section{Results}

\section{Cytological maps of the fourth polytene chromosome}

At the moment, there is no complete detailed cytological map of the fourth chromosome. Small size of the fourth chromosome and bending of its tip, which often tends to bind to the chromocenter ectopically, hinder its spreading on squashed 
chromosome preparations and, therefore, complicate analysis of its banding pattern. Initially, C. Bridges marked the fourth chromosome bands in capital letters A-Z and \&, \&' (Morgan et al. 1934; Fig. 1a). On this map, up to 41 bands can be found taking into account thin dotted bands. On the second variant of

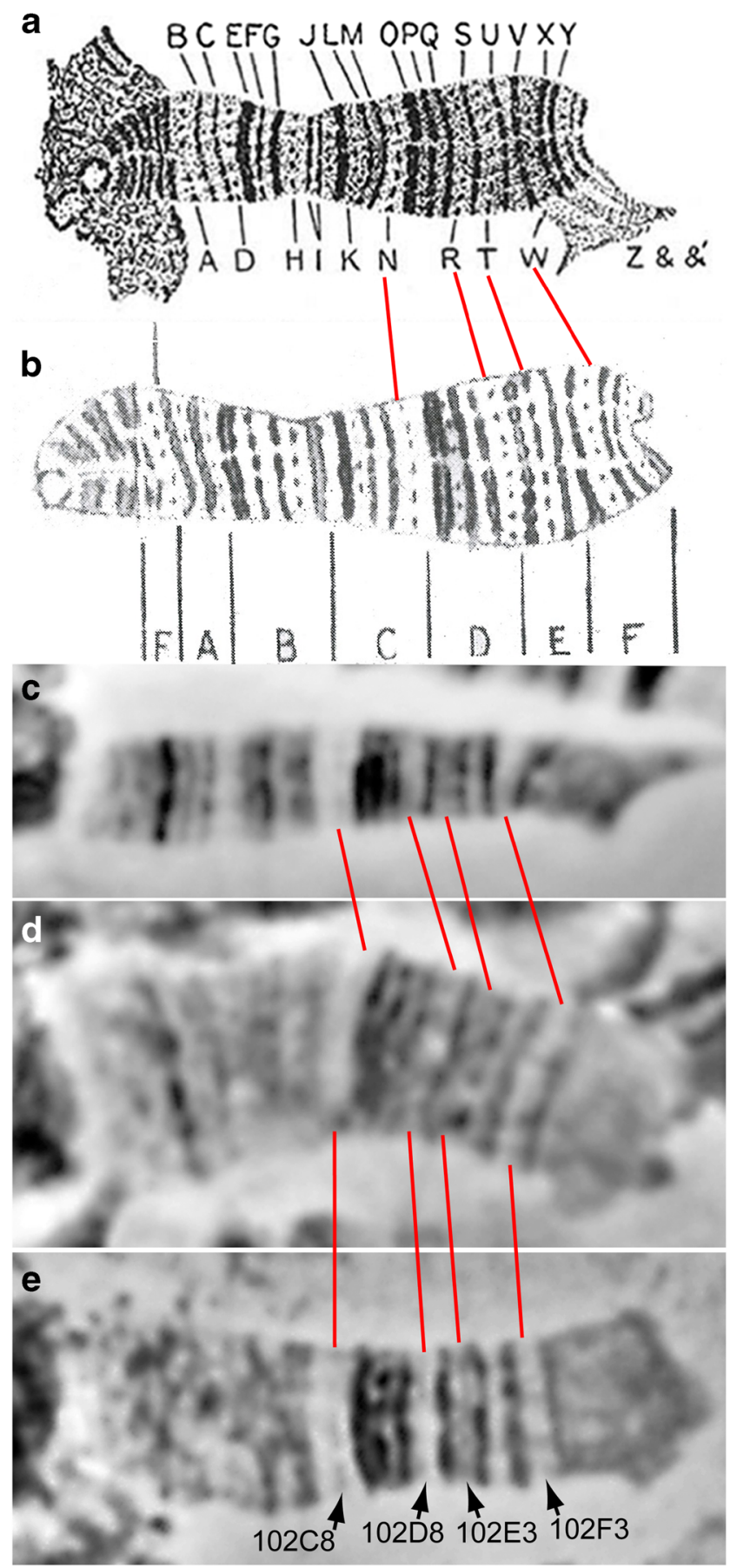

Fig. 1 Thin gray bands in some regions of the fourth chromosome. a Illustration drawn by C. Bridges (Morgan et al. 1934). The drawing is practically the same as in the work by Bridges (1935b). b Drawing of C. Bridges (1935a). c-e Light microscopy of the fourth polytene chromosome. Arrowheads and red lines indicate faint gray bands 102C8, 102D8, 102E3, and 102F3 his map, the polytenized part of the fourth chromosome is divided into 101 and 102 sections and letter subsections (Bridges 1935a; Fig. 1b). Only the section 101F-102F is polytenized (Fig. S1a). Researchers distinguished different numbers of bands of the dot polytene chromosome: from 35, if we count the bands of 101E subsection (Bridges 1935a), to 137 (Slizynski 1944). On polytene chromosome preparations, in fact, you can see much less bands than 137 (Fig. S1b, Fig. $1 \mathrm{c}-\mathrm{e})$; therefore, this number is considered to be unjustifiedly overestimated (Saura et al. 2002). In addition, according to Hochman and Slizynski, the fourth chromosome has a polytenized left arm (King 1975; Slizynski 1944); however, it is unclear whether this structure is indeed a $4 \mathrm{~L}$ chromosome or an ectopically attached portion of another chromosome (Saura et al. 2002). The map by C. Bridges (Bridges 1935a; Fig. $1 \mathrm{~b}$ ) depicts 37 bands of the polytene dot chromosome, if we consider all doublets to be single bands. However, C. Bridges did not complete the creation of a detailed fourth chromosome cytological map; the bands within the subsections did not receive individual numbers. On squashed preparations (Fig. S1b, Fig. 1c-e), one can see within 20-30 bands in the fourth polytene chromosome, depending on the extent of chromosome spreading and the microscopy resolution. Occasionally, the thinnest bands (marked with arrows in Fig. $1 \mathrm{c}-\mathrm{e})$ are seen on well-stretched chromosomes. In the present work, we focused on mapping the polytenized part of the fourth chromosome right arm 101E-102F, and compared our results with the maps by C. Bridges (Fig. 1a, b), electron-microscopic photograph of V. Semeshin (Fig. S1b), and band names from Saura et al. (2002). We made the resulting cytological map of the fourth chromosome based on the map by C. Bridges (1935a), band numbers by Saura et al. (2002), and our mapping efforts (Fig. S1a).

\section{Comparison of the Drosophila fourth chromosome cytological and physical maps}

In this work, we constructed a four-chromatin-state model (ruby, malachite, lazurite, aquamarine) based on the distribution of open chromatin proteins colocalized with CHRIZ for the fourth chromosome of Drosophila. The program algorithm and these chromatin types defined for the remaining chromosomes have been described earlier (Zhimulev et al. 2014; Boldyreva et al. 2017; Materials and methods). Our choice of chromatin state model is substantiated in the Fig. S2 and the Supplementary text 1. It turned out that, in comparison with the large chromosome arms (Zhimulev et al. 2014), the portion of dense ruby chromatin in the fourth chromosome is two times smaller, but malachite and lazurite chromatin types with an intermediate degree of compaction are largely represented (Fig. 2a). This fact is in good agreement with the complex morphological organization of the fourth chromosome with a large number of gray bands. 
a

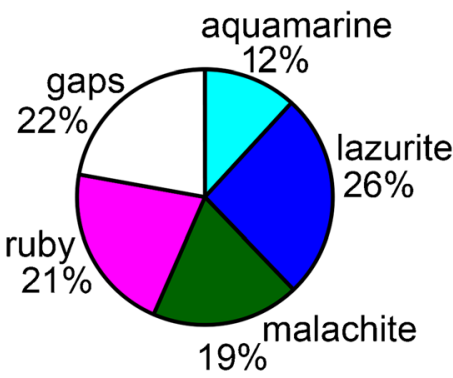

b

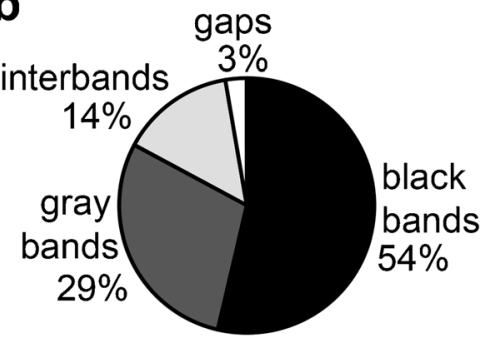

C

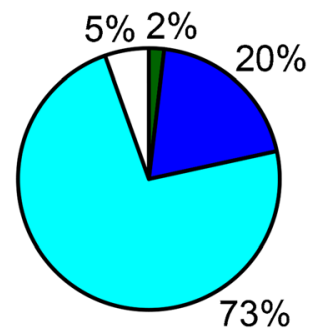

d

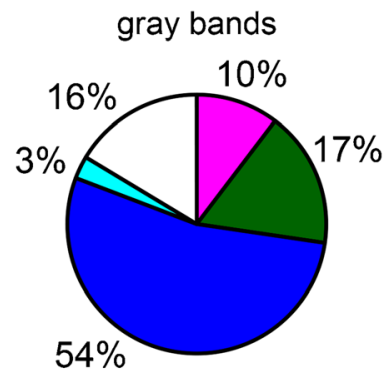

$\mathbf{e}$

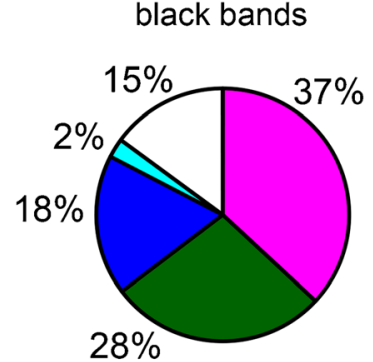

Fig. 2 Chromatin and morphological composition of the fourth chromosome. a The percentage of the four chromatin types in the dot chromosome. Here and further, colors on the diagram correspond to chromatin types and white section corresponds to gaps, where the fourchromatin-state model failed to return a specific value. $\mathbf{b}$ The percentage of bands and interbands in the dot chromosome. Here and further, colors on the diagram correspond to morphological structures and white section corresponds to gaps, which were not included in bands or interbands. $\mathbf{c}$ Chromatin composition of the fourth chromosome interbands. d Chromatin composition of the fourth chromosome gray bands. e Chromatin composition of the fourth chromosome black bands

\section{FISH probe localization on the polytene fourth chromosome}

In order to conduct a fine mapping of D. melanogaster fourth chromosome and connect all its bands and interbands with the physical map of the genome, FISH analysis of 38 DNA probes on polytene chromosomes was carried out (e.g., see Fig. 3). Fifteen of them were selected from the bands predicted by the model of four chromatin states (Zhimulev et al. 2014; Materials and methods), with ruby chromatin corresponding most closely to dense bands (Table S1, Fig. S3, Fig. S4). The initial comparison of the cytological and genomic maps was carried out using "band" samples, as they give a better resolution on FISH preparations. To clarify the boundaries of bands and interbands and to have markers of open chromatin state, 23 additional probes presumably localized in interbands were chosen (Table S2, Fig. S5, Fig. S6, Fig. S7). Mostly, two probes are presented on each preparation. Localization of one of them has already been established in this work, and another one is being mapped on this preparation. Thus, most of the morphological structures of the chromosome studied were covered with the probes. The overall probe hybridization results are plotted on the map by C. Bridges (Fig. 4). The results of these probes localization in the fourth polytene chromosome structures allowed us to relate the fourth chromosome cytological map to definite fragments of the molecular and genetic map (Table S3). A detailed description of the molecular and genetic characteristics of all the fourth chromosome morphological structures is given in the Figures S8-S16 and in the Supplementary text 2.

\section{Chromatin state mapping in the morphological structures of the fourth chromosome}

\section{Molecular characteristics of the 102B3-4-102B5-6 site}

For example, we show the 102B3-4-102B5-6 region of the fourth chromosome (Fig. 5).

The proximal part of the 102B3-4 band is confined by the open chromatin region of $\sim 12.3 \mathrm{~kb}$, represented equally by two aquamarine chromatin fragments surrounding a lazurite fragment. Aquamarine fragments contain the $5^{\prime}$ ends of the genes Syt7, Rad23, Zip102B, and CG32850, arranged "headto-head" in pairs, and CHRIZ and WDS proteins (Fig. 5c, d, $\mathrm{g}$ ). The size of all three fragments is so small that at the cytological level the entire area is perceived as a single 102B1-2/ B3-4 interband (Fig. 5e). The size of the lazurite chromatin inclusion in the interband is $\sim 4 \mathrm{~kb}$, while the smallest fragment size that can form a band detected at the electron microscopy level is $\sim 5 \mathrm{~kb}$ (Semeshin et al. 1989). The FISH probe from the Zip102B gene (lazurite chromatin) marks this interband (Fig. 5e-f, Fig. S5a, Fig. 4b). The 102B3-4 band (Fig. S1) is mainly represented by lazurite chromatin, its total size is $\sim 40 \mathrm{~kb}$, and it has a small malachite chromatin 


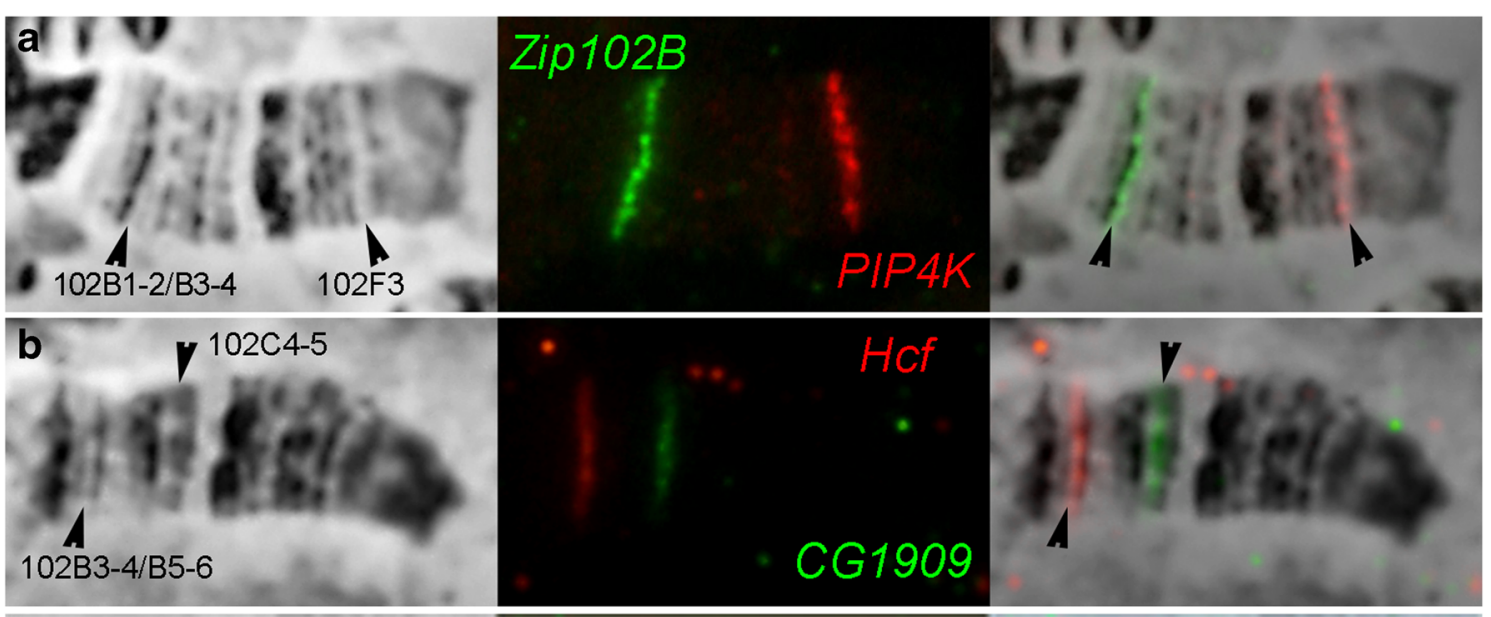

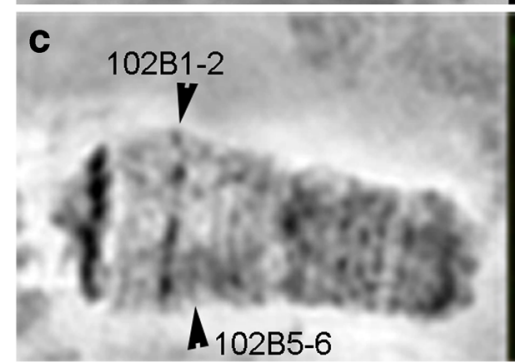

Fig. 3 FISH on the polytene fourth chromosome. From left to right: the phase-contrast microphotograph of the fourth chromosome, combined FISH signals, and their superposition. The red signals correspond to probes labeled with TAMRA fluorochrome, the green ones correspond to probes labeled with fluorescein. The arrowheads indicate the

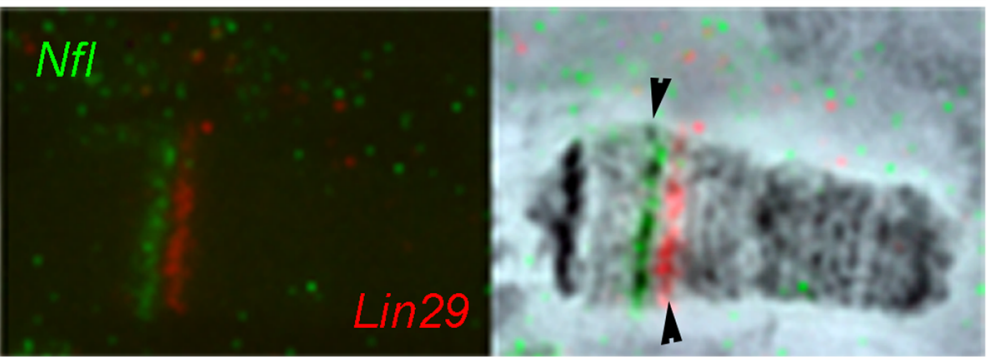

following: a Zip102B probe in the 102B1-2/B3-4 interband, PIP4K probe in the 102F3 band; $\mathbf{b} H c f$ probe in the 102B3-4/B5-6 interband, CG1909 probe in the 102C4-5 band; $\mathbf{c}$ NfI probe in the 102B1-2 band, Lin29 (Dati) probe in the 102B5-6 band.

chromatin, with bordering lazurite chromatin, which usually corresponds to gray bands. Such bands are described in more details in the article by Khoroshko et al. (2018). The distal edge of the 102B5-6 band is flanked by the 102B5-6/B7 interband, the aquamarine chromatin region of $\sim 6 \mathrm{~kb}$ in size with 5' ends of genes Igs and CaMKI located "head-to-head" (Fig. 5c-e).

It is clear that HP1a and POF proteins, specific for the fourth chromosome, are predominantly localized in chromatin types corresponding to interbands and the 102B3-4 gray band and are almost absent in the 102B5-6 black band (Fig. 5d, e, h, i). The binding sites of ORC2 (protein of the replication initiation complex) and the sites of hypersensitivity to DNase I are mainly located in interbands (Fig. 5d, e, j, k).

All other fragments of the fourth chromosome are shown on the Figures S3-S16 and described in detail in the Supplementary text 2; in general, these data correspond to the data described above.

\section{Band and interband chromatin composition of chromosome 4}

Based on the results of mapping, the number of bands (27 pieces, $83 \%$ of the polytenized part length) and interbands (26 pieces, $14 \%$ of the polytenized part length) of the fourth band middle dense area consisting of ruby and malachite 
Fig. 4 The scheme of probe localization in the polytene fourth chromosome, plotted on the map by C. Bridges (Bridges 1935a). a The probes from the bands predicted by the four-chromatinstate model (Zhimulev et al. 2014). b The probes from the interbands predicted by the fourchromatin-state model (Zhimulev et al. 2014).

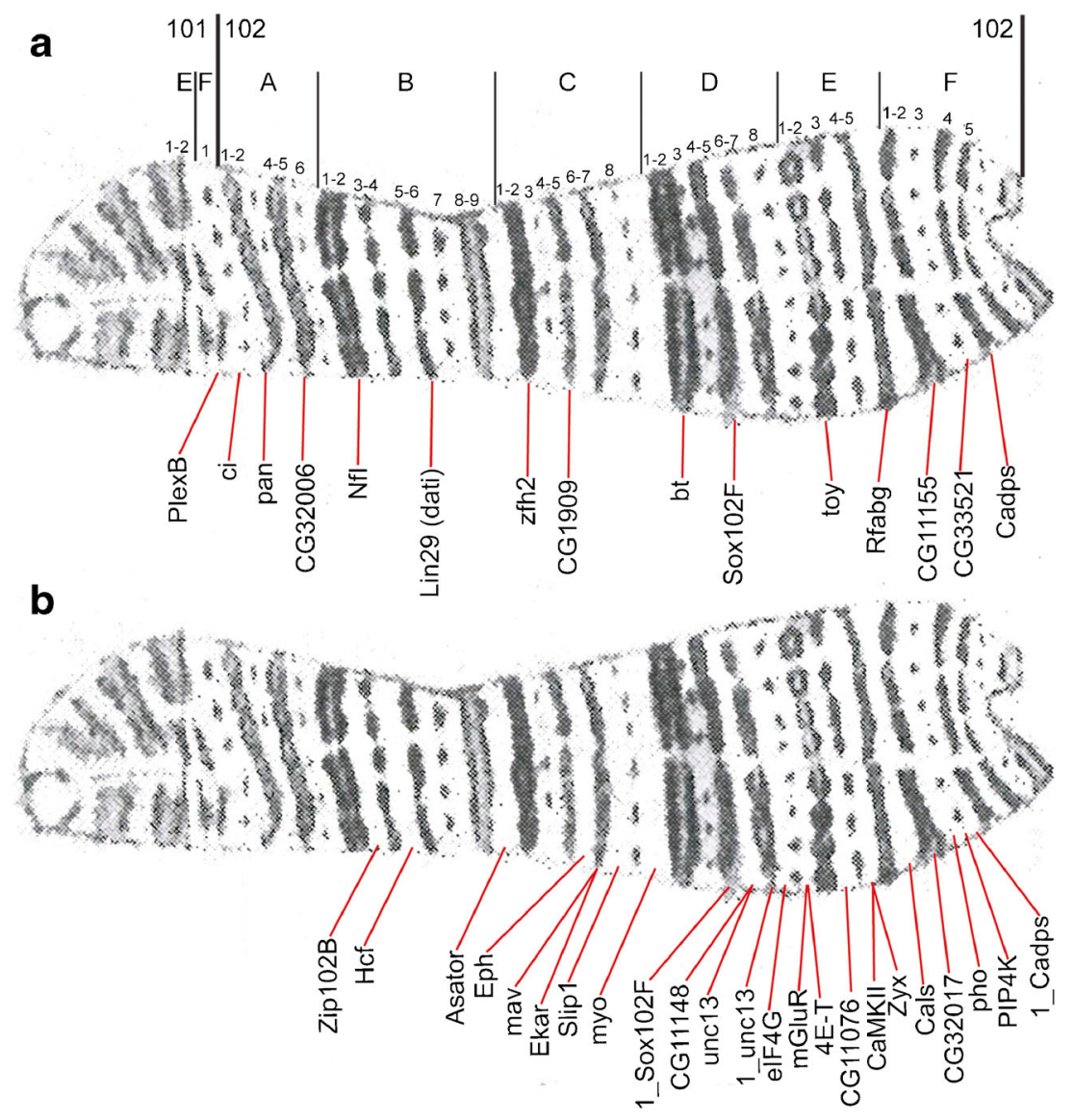

chromosome has been calculated (Fig. 2b). The band size varies from 6.6 to $89 \mathrm{~kb}$ with an average size of $38.41 \mathrm{~kb}$. The interbands are mainly represented by aquamarine chromatin (73\%; Fig. 2c). The number of dense black bands (11 pieces, $54 \%$ of the polytenized part length, Fig. 2b) containing most of the ruby chromatin (occupies $37 \%$ of black bands, Fig. 2e), and more loose gray bands (16 pieces, $29 \%$ of the polytenized part length, Fig. 2b), represented by malachite (17\%) and, to a large extent, lazurite chromatin (54\%, Fig. $2 \mathrm{~d}$ ), is identified. The size of black ruby bands varies from 35.8 to $89 \mathrm{~kb}$ with an average size of $61.04 \mathrm{~kb}$. The size of gray bands varies from 6.6 to $56.8 \mathrm{~kb}$ with an average size of $22.86 \mathrm{~kb}$. Black ruby bands are as follows: 101E1-2, 102B1-2, 102B5-6, 102C1-2, 102D1-2, 102D4-5, 102D6-7, 102E1-2, 102E4-5, 102F1-2, and 102F4,5. Gray bands are as follows: 101F1, 102A1-2, 102A4-5, 102A6, 102A6', 102A6", 102B34, 102B7, 102B8-9, 102C3, 102C4-5, 102C6-7, 102C8, 102D8, 102E3, and 102F3.

\section{Exceptions in the chromatin composition of bands and interbands}

A part of the interbands ( 8 of 26) has a complex structure according to the model of four chromatin types. In addition to aquamarine chromatin, they contain small, invisible even with electron microscopy in the form of band insertions of lazurite (seven inclusions) and malachite chromatin (two inclusions). Inclusions of lazurite chromatin correspond to structural parts of ubiquitously active genes (according to FlyBase). One of the malachite chromatin inclusions corresponds to the gene intron, and the other corresponds to the intergenic interval. The size of the lazurite inclusions varies from 2.8 to $7.2 \mathrm{~kb}$, and the average value is $5.3 \mathrm{~kb}$. Malachite inclusion measurements are 1.6 and $1.8 \mathrm{~kb}$. All these inclusions are small in size. According to V. Semeshin, at the limit of electron microscopy resolution, in some cases, it is possible to see structures of at least $5 \mathrm{~kb}$ in size as individual bands (Semeshin et al. 1989).

A part of the bands (13 of 27 , including 8 black bands: 102B5-6, 102C1-2, 102D1-2, 102D4-5, 102D6-7, 102E1-2, 102E4-5, 102F1-2, and 5 gray bands: 102A1-2, 102A4-5, 102С6-7, 102C8, и 102F3) has a complex structure and contains small inclusions of aquamarine chromatin according to the model of four chromatin types. In total, we found 21 inclusions, the size of which varies from 0.4 to $3.8 \mathrm{~kb}$ with an average value of $1.2 \mathrm{~kb}$. Ten aquamarine inclusions in the bands contain $5^{\prime}$ ends of the genes that, basically, do not work in larval salivary glands. The exception is the fragment of aquamarine in the 102F3 band at the distal end of the fourth chromosome, which has all the features of the interband; 
$a$

b

c

d

g

CHRIZ_BR.S2

CHRIZ_WR.S2

CHRIZ BR.KC

CHRIZ_BR.BG3

CHRIZ_BR.Clone 8

CHRIZ_BR.14-16hORemb WDS_Q2691.S2

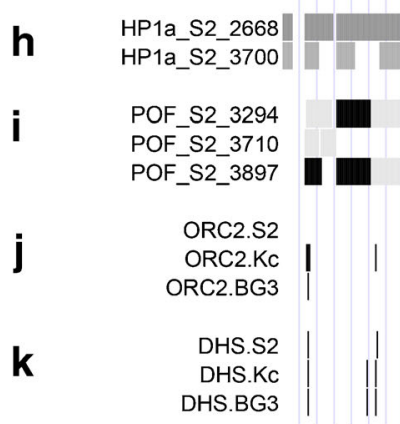

Fig. 5 Molecular, genetic, and cytological organization of the fourth chromosome 102B3-4-102B5-6 site: a the scale (kb); b the genomic coordinates (bp); $\mathbf{c}$ the genes (denoted by a curly bracket); $\mathbf{d}$ the fourchromatin-state model; the colors correspond to the type names (Zhimulev et al. 2014; Boldyreva et al. 2017); e the scheme of band and interband localization relative to the genomic coordinates. The band names are denoted. The black ruby-containing bands are shown in black rectangles, the gray bands are shown in dark gray rectangles, and the

however, we assume that, according to the map by C. Bridges, electron and light microscopy, this area contains only one gray band, 102F3. The identification of the interband-specific aquamarine state within a band by the model of four chromatin types, based on cell culture data, may be related to the genome plasticity, i.e., the presence of genes that are expressed and form an active interband in diploid tissues, but do not work in salivary gland polytene chromosomes and do not form an interband there. Five aquamarine inclusions in the bands contain intergenic spacers, and six ones contain $3^{\prime}$ ends of genes or their coding parts. It has been shown earlier that small aquamarine chromatin inclusions in large ruby-containing bands are enriched in enhancers and insulators (Khoroshko et al. 2016).

It was shown that a part of large black intercalary heterochromatin bands containing ruby chromatin has lazurite chromatin at the borders, which usually corresponds to gray bands containing structural parts of ubiquitously active genes. The band size is substantially increased due to the integration of the middle dense band region of ruby and malachite chromatin with bordering lazurite chromatin. Such bands are described in more detail by Khoroshko et al. (2018). In the fourth chromosome, one or both borders of 10 out of 13 ruby-containing bands are represented by lazurite chromatin.

A detailed description of all bands and interbands is provided in the Supplementary text 2.

\section{Genetic content of the fourth chromosome bands and interbands}

We determined the genetic content of the fourth chromosome cytological structures (Table S3). It turned out that all interbands contain 5'untranslated regions of genes, with some interbands ( 6 pieces) containing genes completely localized in these open chromatin domains (Fig. 6a). Gray bands mainly contain the structural parts of genes with 5'regulatory regions located in flanking interbands (11 of 16 bands). The exceptions are the 102D8 band, which contains the whole gene, and the 102A4-5, 102C4-5, 102C6-7, and 102F3 polygenic bands (Table S3, Fig. 6a). Black bands are mainly polygenic (8 of 11 bands). The exceptions are the 102D6-7 band and the first (101E1-2) and the last $(102 \mathrm{~F} 4,5)$ bands containing genes 
Fig. 6 Genetic organization of the fourth chromosome domains and gene expression. a Enrichment of various parts of genes in the structures of the fourth chromosome. The $Y$-axis indicates the percentage $(\%)$ of each category. b The number of larval tissues (on the $Y$-axis) in which genes of the fourth chromosome are expressed (Chintapalli et al. 2007). c The number of adult fly tissues (on the $Y$-axis) in which genes of the fourth chromosome are expressed (Chintapalli et al. 2007).

\section{a}

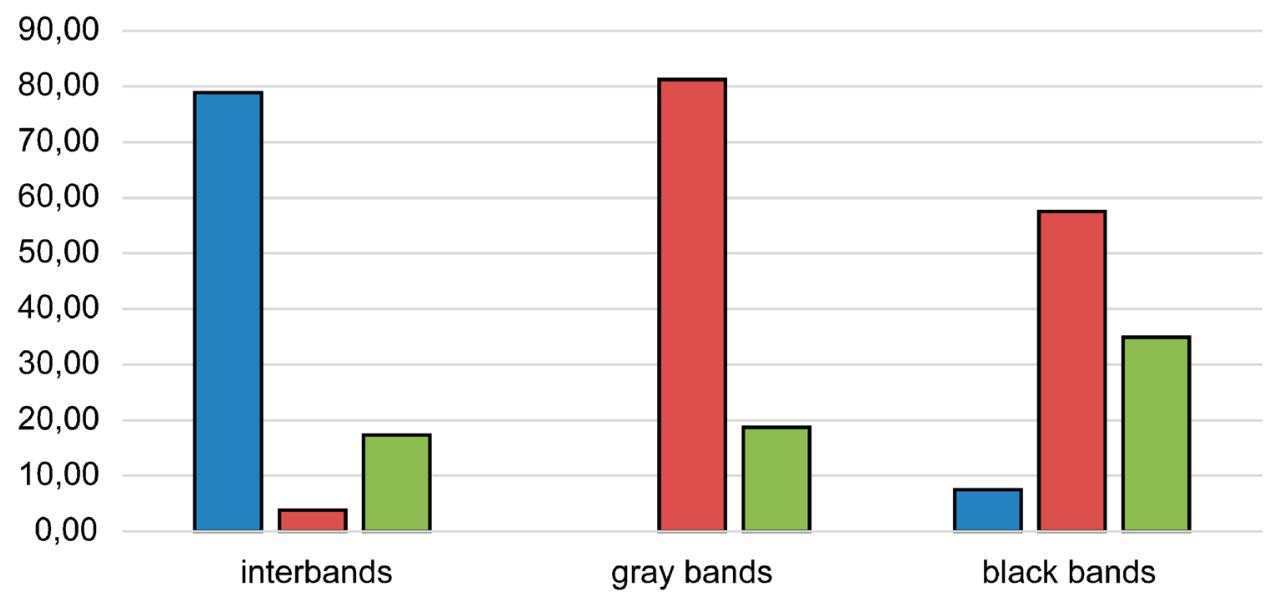

घ'UTR $\square$ coding part of gene $\square$ whole gene

b

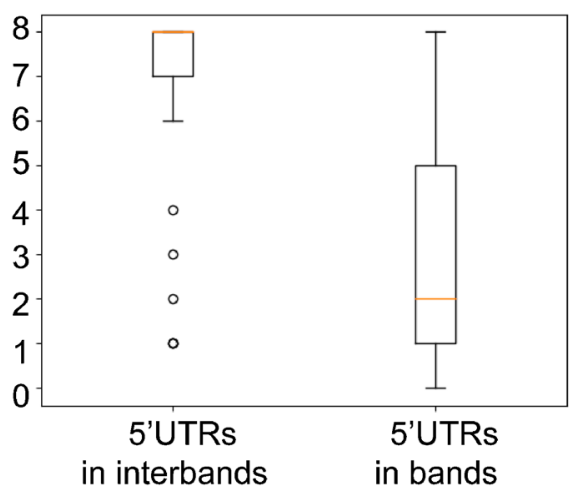

C

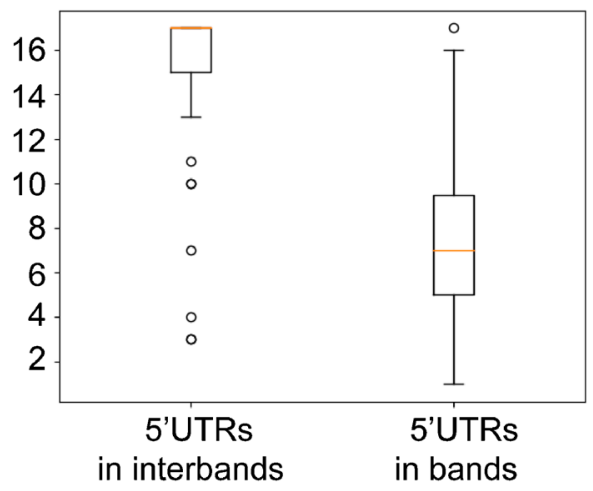

starting in the flanking interbands (Table S3, Fig. 6a). Black bands are enriched with coding parts of genes due to lazurite chromatin attachment to the edges of black bands. Such structures are described in more detail in the Supplementary text 2 and in the work by Khoroshko et al. (2018).

We compared the expression of genes whose 5' regulatory regions are located in cytologically mapped interbands, with genes localized in gray and black bands of the fourth chromosome. It turned out that genes that begin in the open chromatin of interbands are on average expressed in more tissues of larvae (Fig. 6b) and adult flies (Fig. 6c) compared to genes, 5'UTRs of which are located in gray and black bands. Similar data have been obtained previously for the complete Drosophila genome (Zhimulev et al. 2014).

\section{Aquamarine and lazurite chromatin types of the fourth chromosome are enriched with POF and HP1a proteins}

A statistical analysis of the differences in the distribution of proteins in the four chromatin types of the fourth chromosome and the rest of the Drosophila genome was performed using the Mann-Whitney $U$ test. Figure S17 shows all proteins whose proportion in certain chromatin types of the fourth chromosome significantly differs from this value for the complete genome. Table 1 indicates proteins that have a significant difference between the medians of the proportions occupied by them in the corresponding type of chromatin of the fourth chromosome and the rest of the Drosophila genome.

Aquamarine and lazurite active chromatin types of the fourth chromosome that correspond to the regulatory regions and the bodies of housekeeping genes in the Drosophila genome, the interbands and loose gray bands respectively (Zhimulev et al. 2014), are enriched with POF protein unique for this chromosome (Larsson et al. 2001). In addition, these two types of chromatin are enriched with the heterochromatin protein HP1a, and the HP2 protein colocalized with it. Lazurite chromatin of the fourth chromosome relative to the genome lazurite chromatin is enriched with the SU(VAR)3-9 histone methyltransferase, which marks inactive chromatin with $\mathrm{H} 3 \mathrm{~K} 9$ methylation. Aquamarine and lazurite chromatin of the fourth chromosome are depleted with a large number of histone modifications associated with active transcription, and with open chromatin proteins. Aquamarine chromatin of the 
Table 1 Enrichment and depletion of chromatin types of the fourth chromosome with proteins and histone modifications (modENCODE data, S2 cells) in comparison with the chromatin types of the rest of the Drosophila genome

\begin{tabular}{lll}
\hline $\begin{array}{l}\text { Chromatin } \\
\text { type }\end{array}$ & $\begin{array}{l}\text { Enrichment with proteins and histone } \\
\text { modifications in the fourth chromosome }\end{array}$ & $\begin{array}{l}\text { Depletion with proteins and histone modifications } \\
\text { in the fourth chromosome }\end{array}$ \\
\hline Aquamarine & HP1a, HP2, POF & $\begin{array}{r}\text { H2Bubiq, H3K4me1, H3K4me2, H3K79me2, } \\
\text { H3K9ac, H4K16ac, H4K8ac, ISWI, MOF, } \\
\text { MRG15, RNA-pol II, WDS }\end{array}$ \\
Lazurite & HP1a, HP2, POF, SU(VAR)3-9 & H2AV, H2Bubiq, H3K27me1, H3K4me1, \\
& & H3K4me2, H3K79me1, H3K79me2, \\
& H3K79me3, H3K9ac, H4K16ac, CHRIZ, \\
& JIL1, MRG15, RNA-pol II \\
Malachite & H2AV, HP1a & \\
Ruby & H3K9me2, SU(VAR)3-9 & \\
\end{tabular}

fourth chromosome relative to the genome aquamarine chromatin is also depleted with the ISWI remodeling protein associated with a higher order chromatin structure involving H1 histone (Deuring et al. 2000; Corona et al. 2007).

Malachite chromatin of the fourth chromosome in comparison with the malachite chromatin of the rest of the genome is enriched with the heterochromatin protein HPla and the histone variant $\mathrm{H} 2 \mathrm{AV}$. The phosphorylated form of $\mathrm{H} 2 \mathrm{AV}$ $(\gamma \mathrm{H} 2 \mathrm{AV})$ is associated with double-stranded DNA breaks, possibly caused by underreplication (Madigan et al. 2002; Mehrotra and McKim 2006; Andreyeva et al. 2008). As it has been shown earlier, this chromatin type mostly corresponds to intergenic intervals and introns of genes, and also it is a part of active gene long introns forming thin gray bands of polytene chromosomes (Zhimulev et al. 2016; Boldyreva et al. 2017).

Transcriptionally inactive ruby chromatin of the fourth chromosome is enriched with H3K9me2 and SU(VAR)3-9 marks of repressed chromatin relative to the ruby chromatin of the rest of the genome.

\section{Distribution of ORC2, HP1a, POF, CHRIZ, H3K27me3, and SUUR proteins in the fourth chromosome of $D$. melanogaster}

We studied the distribution of the replication complex protein ORC2 (modENCODE data). The results suggest that the ORC2 binding sites in the fourth chromosome are mainly localized in aquamarine chromatin, which corresponds to the interbands (Fig. 7a, b). This is in good agreement with the whole genome data (Zhimulev et al. 2014). The ORC2 distribution density in each morphological structure of the fourth chromosome is shown in Figure S18. A more detailed analysis showed that ORC2 in the fourth chromosome has a binding peak within $400 \mathrm{bp}$ around the beginning of genes, whose 5' UTRs lie in the interbands (Fig. 7c).

The distribution of HP1 a and POF proteins (modENCODE data), which are responsible for the special epigenetic status of the fourth chromosome, was studied in more detail. Analysis of the chromatin immunoprecipitation data on the studied proteins in S2 cells showed that they are located predominantly in domains corresponding to interbands and gray bands of the fourth chromosome (Fig. 8a). Relative to the model of four chromatin types, these proteins are localized in lazurite chromatin (Fig. 8b), which corresponds to the coding parts of genes (Fig. 6a) and, generally, gray bands (Fig. 2d), and in aquamarine chromatin (Fig. 8b), which corresponds to promoters of ubiquitously active genes (Fig. 6a) and interbands (Fig. 2c).

In addition, we studied the distribution of POF protein in salivary gland cells of Drosophila larvae. The analysis of chromatin immunoprecipitation results of this protein specific for the fourth chromosome (Lundberg et al. 2013a; Johansson and Larsson 2014) showed that, as in S2 cells, POF predominantly localizes in lazurite and aquamarine chromatin types (Fig. 9a) and in the corresponding gray bands and interbands of the fourth chromosome (Fig. 9b). POF is completely absent in the highly compact ruby chromatin. Localization of POF in black bands is explained by the fact that these bands in the fourth chromosome contain not only ruby chromatin but also a rather large proportion of malachite and lazurite chromatin (Fig. 2e).

Thus, protein POF is localized in some sites of open chromatin of the fourth chromosome. Another open chromatin protein is CHRIZ, which specifically decorates the interbands of polytene chromosomes (Gortchakov et al. 2005). We aimed to find out how are these proteins located relative to each other. To achieve this goal, we performed coimmunostaining of Drosophila polytene chromosomes with POF and CHRIZ antibodies (Fig. 10). We obtained a very stretched well-spread polytene fourth chromosome using SuUR ${ }^{E S}$ Su(var)3-9 $9^{06}$ double mutant flies with suppressed underreplication (Fig. 10a). The protein CHRIZ was expected to occupy all interbands of the fourth chromosome, but it appeared to occupy almost all of them except one (Fig. 10b, c). The 102B5-6/B7 interband remains empty (marked with an asterisk in Fig. 10). In addition, CHRIZ antibodies also paint the telomeric loose gray tip of the fourth chromosome. The protein POF is localized in some interbands and gray bands of the fourth chromosome, and never in black bands (17 signals 
Fig. 7 ORC2 protein distribution (modENCODE data): $\mathbf{a}$ in the cytological structures of the fourth chromosome, with the first column corresponding to all the bands of the fourth chromosome, and further - as shown in Fig. $2 \mathrm{~b}$; $\mathbf{b}$ in the four chromatin types of the fourth chromosome. The ordinate shows the density in pcs/ $\mathrm{kb}$. $\mathrm{C}$ ORC2 protein distribution (modENCODE data) in S2 cells relative to genes beginning in the interbands of the fourth chromosome. The interbands are divided into fragments of $200 \mathrm{bp}$ from the beginning of the gene towards the intergenic spacer (s1s5) and towards the structural part of the gene ( $\mathrm{g} 1-\mathrm{g} 5)$; the genes are aligned with respect to the beginning. The graph shows five fragments in each direction. The ordinate shows the total number of sites in these fragments, normalized by the number of the gene transcripts. The red arrow shows the start and direction of the genes. a

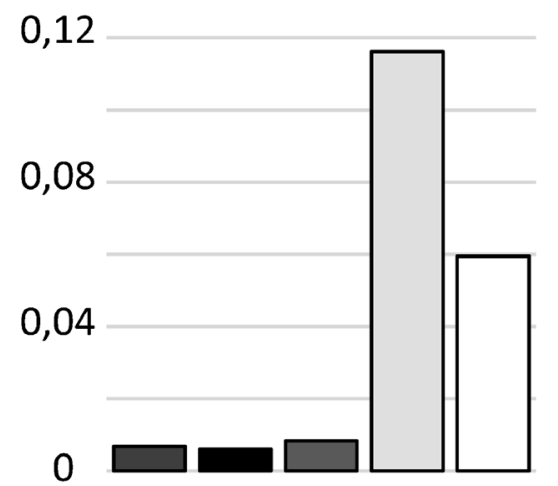

b

0,16

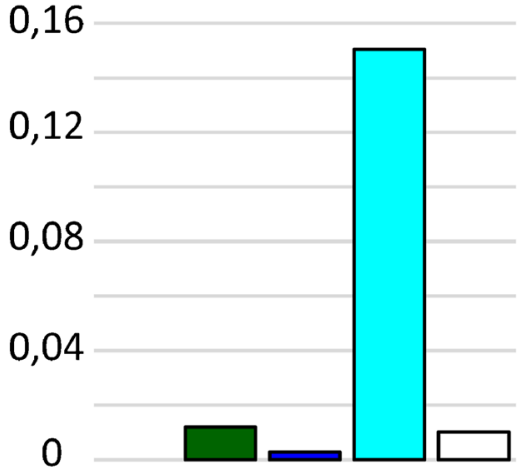

C

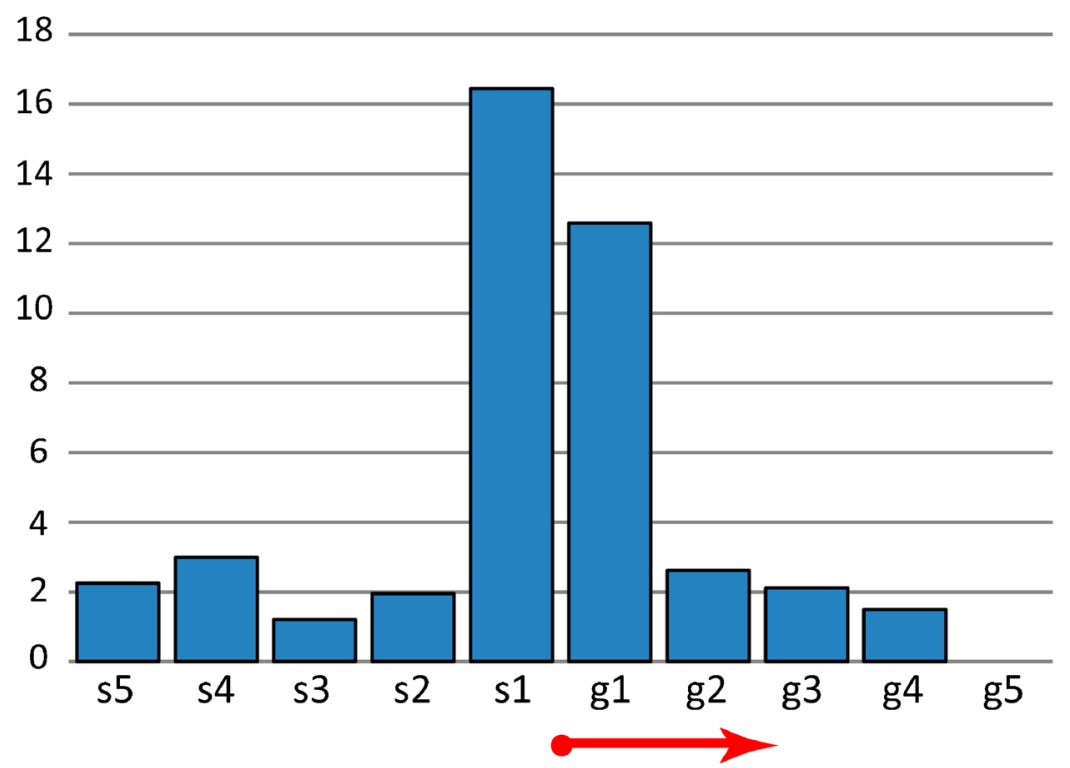

in total; Fig. 10d, e). Interestingly, the 102B5-6/B7 interband also lacks POF binding. However, this issue is yet to be addressed. The protein POF only partially colocalizes with CHRIZ (Fig. 10f, g). Both of the studied proteins are absent from pericentric part of the fourth chromosome. Thus, at the cytological level, the proteins POF and CHRIZ occupy open chromatin sites of the fourth chromosome and only partially colocalize. This observation is supported by the molecular data, since it has been shown that POF specifically binds to genes with a strong exon preference (Johansson et al. 2007b), and the exons of active genes correspond to lazurite chromatin at the whole genome level (Zykova et al. 2018), whereas CHRIZ is generally associated with the promoters of housekeeping genes in aquamarine chromatin (Zhimulev et al. 2014; Zykova et al. 2018).

Trimethylated histone $\mathrm{H} 3$ at the 27th lysine is the epigenetic mark of repressed chromatin. It is associated with Polycomb-dependent heterochromatin. We analyzed the distribution of this H3K27 modification obtained in the DamID experiment in Drosophila salivary gland cells (Sher et al. 2012; Fig. S19i) relative to the model of four chromatin types (Zhimulev et al. 2014; Materials and methods) and in the morphological structures of the fourth chromosome. It turned out that H3K27me3 is enriched only in dense ruby chromatin (Fig. 11a), which generally corresponds to black bands. When considering the distribution of this modification in the morphological structures, it is shown that in black bands, the median value is maximal in comparison with the remaining morphological structures; however, it is about 0 (Fig. 11b). This is due to the fact that ruby chromatin is only about a third of the total length of black bands, and the median value of H3K27me3 enrichment is affected by all other types of chromatin in the composition of black bands (Fig. 2e).

Analyzing the protein composition of the fourth chromosome, we found that the data on the distribution of the Supressor of Underreplication protein (SUUR) in the fourth chromosome of $D$. melanogaster, obtained earlier by using the DamID method in larval salivary glands (Maksimov et al. 


\section{a}

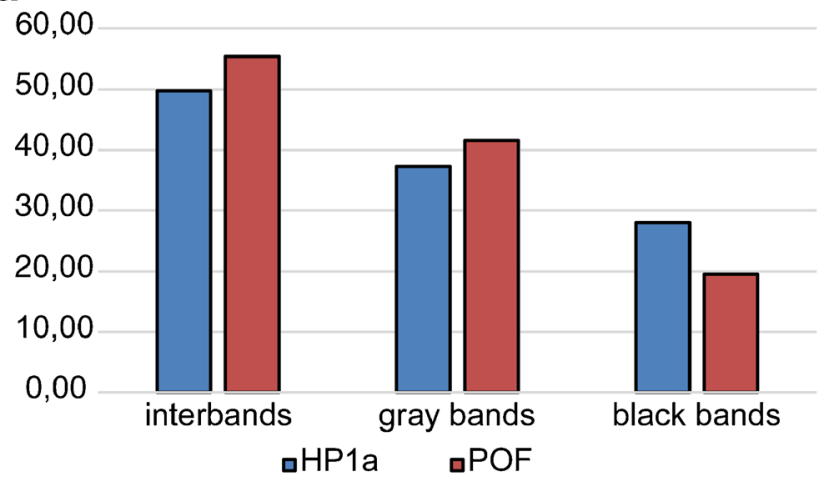

b

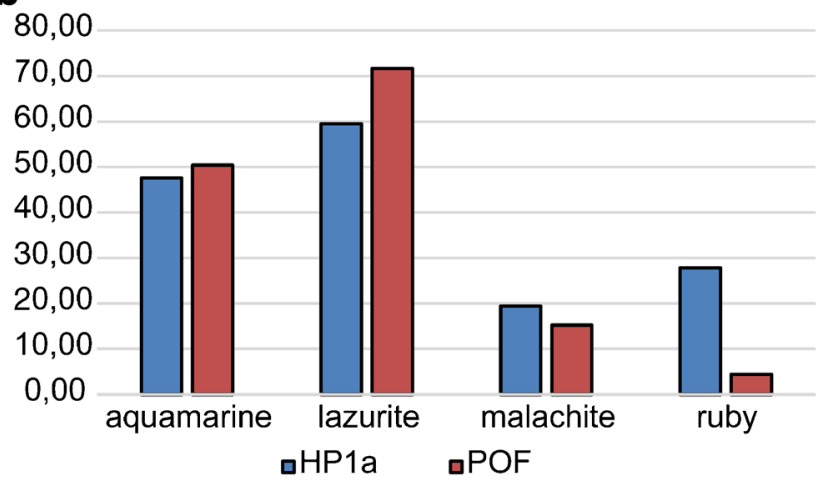

Fig. 8 Distribution of HP1a and POF proteins (modENCODE data) in the fourth chromosome in $\mathrm{S} 2$ cells. The diagram on the $Y$-axis shows the proportions (\%) of the total length of morphological structures (a) and chromatin domains (b) occupied by HP1a and POF.

2014; Posukh et al. 2017), in cell cultures (Filion et al. 2010; Maksimov et al. 2014), embryos, and brain (Maksimov et al. 2014) do not correspond to the immunolocalization data on polytene chromosomes (Fig. S19k-p, Fig. 12). The data on salivary gland chromosomes (Posukh et al. 2017) reveal almost continuous signal of SUUR binding on the fourth chromosome (Fig. S191). Although SUUR binding in Drosophila chromosomes is dynamic and varies depending on the stage of replication (Kolesnikova et al. 2013), none of the stages shows continuous painting of the fourth polytene chromosome. The immunostaining reveals up to four discrete signals of this protein binding: near the centromere and telomere regions, and two bands in 102B and 102D regions (Fig. 12), as it has been reported earlier (Zhimulev et al. 2003).

\section{Specificity of mobile element distribution in the fourth chromosome}

\section{P-element distribution}

The distribution of $P$-transposon insertions into morphological structures and various chromatin types of the fourth chromosome was studied (107 insertions in total). These mobile genetic elements are distributed not randomly. In the fourth chromosome, $P$ - elements are predominantly embedded in interbands and aquamarine chromatin maximally corresponding to these structures (Fig. 13a, b) as it has previously been shown for the whole Drosophila genome (Zhimulev et al. 2014). The density of the insertions in each structure of the fourth chromosome is shown in Figure S20. A more detailed analysis showed that the maximum number of $P$-transposons in the fourth chromosome is located within $400 \mathrm{bp}$ around the 5'UTRs of genes in interbands and aquamarine chromatin corresponding to them (Fig. 13c).

\section{0 distribution}

In addition, the specificity of the 1360 element distribution in the fourth chromosome of $D$. melanogaster was determined. The 1360 element exhibits strain-specific location in the euchromatic parts of the chromosomes, but constant heavy labeling of the 12E1-2, 42B1-3, 52A1-2, 62A1-2, 75B, and 82C1-3 regions, chromosome bases, the chromocenter, and numerous sites of the fourth chromosome (Kholodilov et al. 1988). Sequence analysis revealed 37-bp terminal inverted repeats, flanked by 6-bp direct repeats, and two inverted repeats of $15 \mathrm{bp}$ in the central part, capable of hairpin formation. This
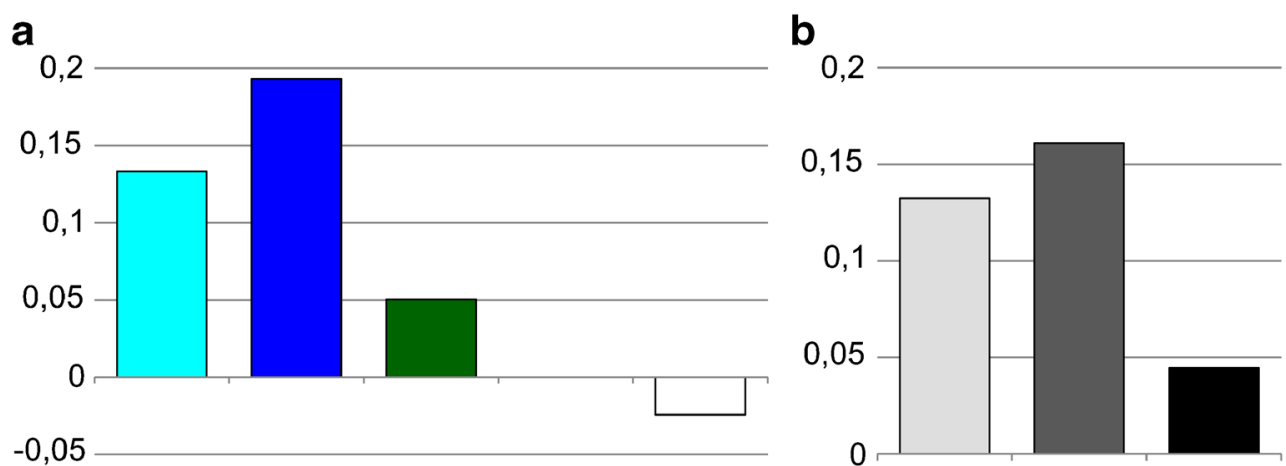

Fig. 9 Distribution of POF protein in the fourth chromosome in the salivary gland cells of Drosophila larvae (Lundberg et al. 2013a; Johansson and Larsson 2014) a relative to the bands and interbands and

b relative to the chromatin domains of four types (Zhimulev et al. 2014; Materials and methods). The value of the median of the ChIP profile peaks for each type of domains is shown on the $Y$-axis. 


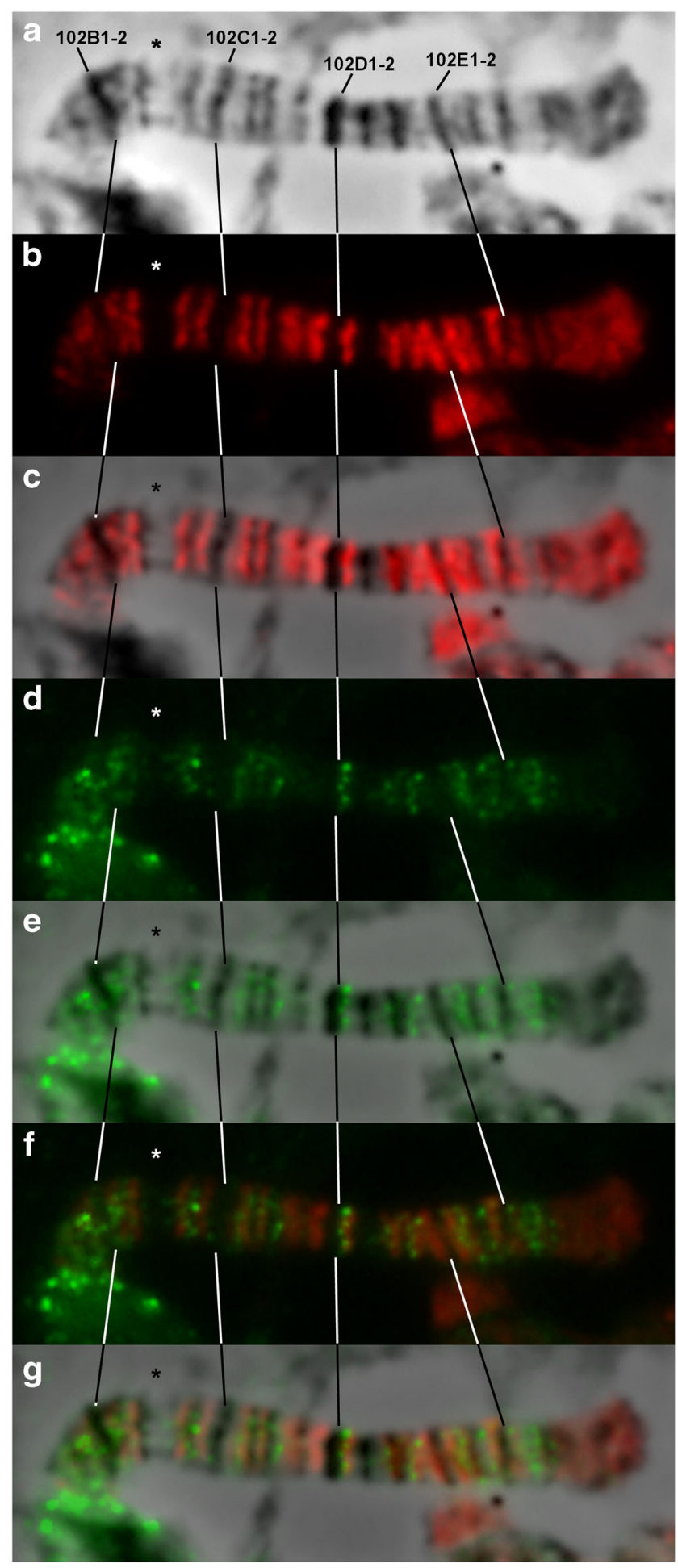

Fig. 10 POF and CHRIZ localization on the polytene fourth chromosome of D. melanogaster. a Phase contrast image of the fourth chromosome. b CHRIZ staining. Antibodies are the same as in Gortchakov et al. (2005). c Merged phase contrast and CHRIZ staining. d POF staining. Antibodies are the same as in Larsson et al. (2001). e Merged phase contrast and POF staining. f Merged CHRIZ and POF staining. g Merged phase contrast, CHRIZ, and POF staining. The arrow indicates the 102B5-6/B7 interband where no POF and CHRIZ binding was observed. fragment called "element 1360 " has high A-T content and significant number of short nucleotide tracts. It has sequences with homology to the $F B$ - and $P$-elements (as a rule, poly(A) and poly(T) tracts, characteristic of these transposable elements). The 1360 element belongs to the Hoppel family (Reiss et al. 2003) and is a particular class of these dispersed and deleted sequences (Kholodilov et al. 1988; Kurenova et al. 1990). The 1360 element is entirely encompassed within the 3.4-kb Hoppel element (Reiss et al. 2003).

In total, there are 59 remnants of the 1360 element (modENCODE data) in the fourth chromosome; four of them were excluded from the analysis, since they completely overlapped with other remnants. The total length of 1360 remnants in the Drosophila fourth chromosome is 49,974 nucleotides. It is shown that the 1360 element is distributed in the fourth chromosome of Drosophila not in a random manner. In contrast to $P$-elements that tend to integrate into open chromatin, 1360 element is predominantly located in the fourth chromosome bands and ruby, malachite, and lazurite chromatin corresponding to bands. The distribution of 1360 element in the cytological structures and different types of chromatin of the fourth chromosome is shown (Fig. 13d, e).

\section{Distribution of the DNasel hypersensitivity sites}

We show that the DHS in the fourth chromosome predominantly localize in aquamarine chromatin corresponding to interbands, which agrees with the data obtained for the rest chromosomes (Fig. 14a, b; Zhimulev et al. 2014). The density of DHS in each morphological structure of the fourth chromosome is shown (Fig. S21). A more detailed analysis showed that DHS in the fourth chromosome are predominantly located within $200 \mathrm{bp}$ upstream the $5^{\prime}$ UTRs of genes in the interbands (Fig. 14c).

\section{Discussion}

The correlation of the Drosophila genome physical map and the large amount of data on gene localization, histone modifications, chromatin proteins, and various regulatory sites with detailed cytological maps of polytene chromosomes reflecting the structural organization of the genome in the interphase nucleus allows us to understand the patterns of genome functioning and their relationship to chromosome morphology. This work was devoted to such investigation of the Drosophila fourth chromosome.

The fourth chromosome was chosen as the object of study, since it has a number of unique properties. It combines the properties of eu- and heterochromatin. High density of active genes corresponding to euchromatin is mixed with the absence of recombination and, as a consequence, high density of repeated DNA. In particular, the fourth chromosome comprises the 1360 element, which, in addition, is present only in 
Fig. 11 The distribution of H3K27me3 in the Drosophila larvae salivary gland cells (Sher et al. 2012). $\mathbf{a}$ in the four chromatin types of the fourth chromosome, and $\mathbf{b}$ in the bands and interbands of the fourth chromosome. The median value of the distribution profile peaks is shown on the $Y$-axis a

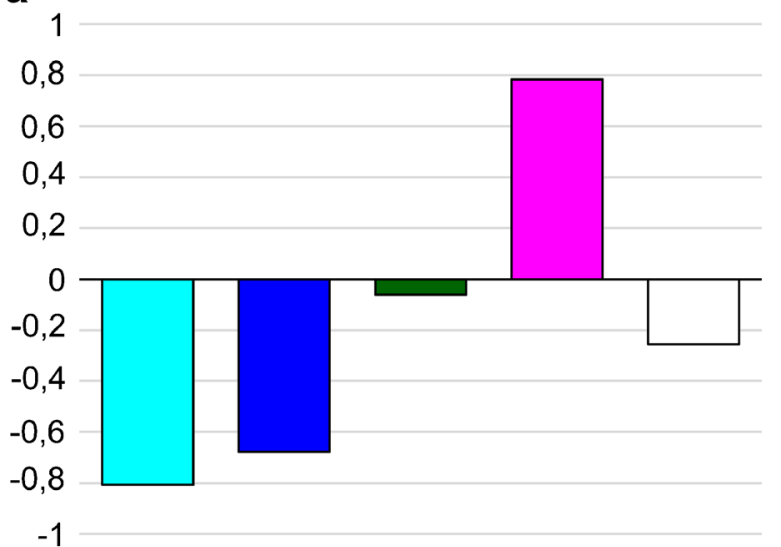

b

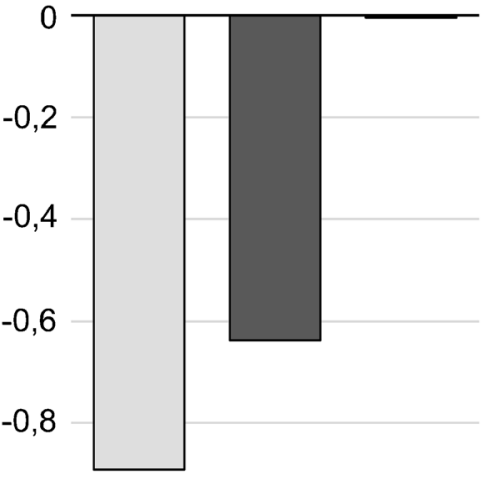

$-1$ several genome sites (Kholodilov et al. 1988). Chromatin of the fourth chromosome is characterized by $\mathrm{H} 3 \mathrm{~K} 9$ methylation and a typical heterochromatin protein HP1 recognizing this epigenetic mark. Herewith, methylation of H3K9 is introduced by dSETDB1, histone methyltransferase specific for this chromosome. Moreover, POF protein specifically stains the fourth chromosome on squashed preparations. In addition, small size, a large number of gray bands, and frequent ectopic contacts of its distal tip with the chromocenter complicate microscopic analysis of the fourth polytene chromosome, so at this time there is no detailed, universally recognized cytological map.

In this work, we refined the cytological map of the fourth chromosome and superposed its bands and interbands with the genomic coordinates using the FISH analysis and the fourchromatin-state model (Zhimulev et al. 2014; Materials and methods). This allowed us to determine some molecular characteristics of the cytological structures of the studied chromosome using open access databases.

The unique feature of the four-chromatin-state model is that it reveals the aquamarine chromatin type that is enriched with interband-specific proteins, and it is this chromatin type which allows matching cytological and molecular chromosome maps (Zhimulev et al. 2014; Boldyreva et al. 2017). The fact is that the interbands correspond to the boundaries of topologically associated domains (Stadler et al. 2017). Other chromatin state models that yield epigenetic domains based on clustering chromatin proteins do not distinguish a separate "interband" chromatin type (Kolesnikova 2018; Kolesnikova et al. 2018). Thus, the four-chromatin-state model designates the boundaries of physical but not epigenetic domains that not always coincide (Hou et al. 2012).
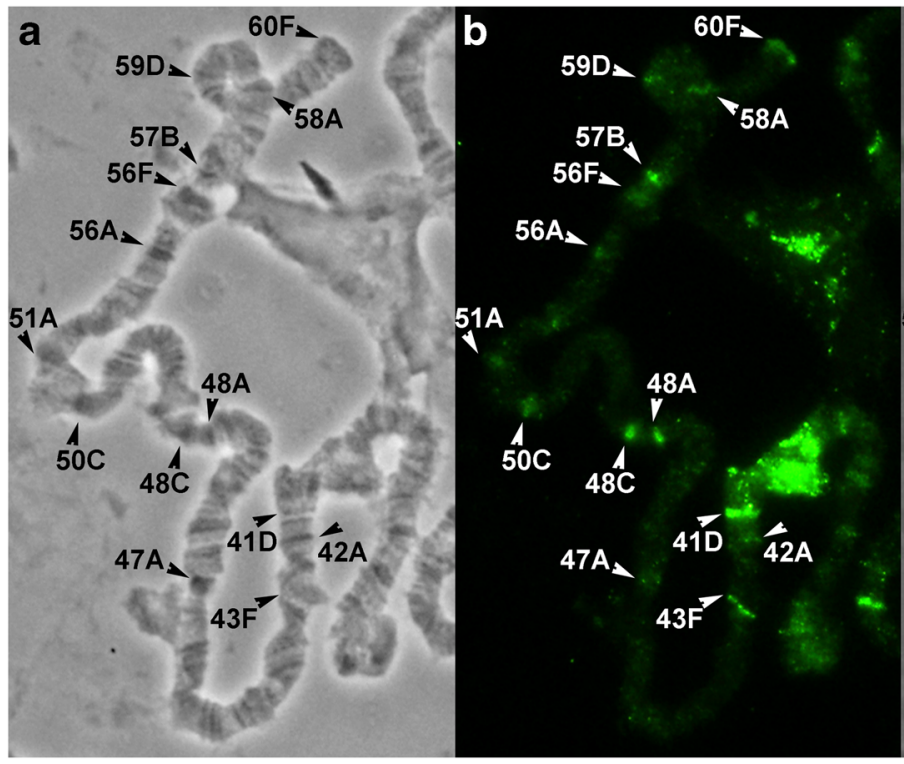

Fig. 12 SUUR localization in polytene chromosomes. The picture is kindly provided by T. D. Kolesnikova. a Phase contrast of the $2 \mathrm{R}$ chromosome. b SUUR localization in $2 \mathrm{R}$ chromosome. c Merged image of phase contrast and SUUR localization in the $2 \mathrm{R}$ chromosome

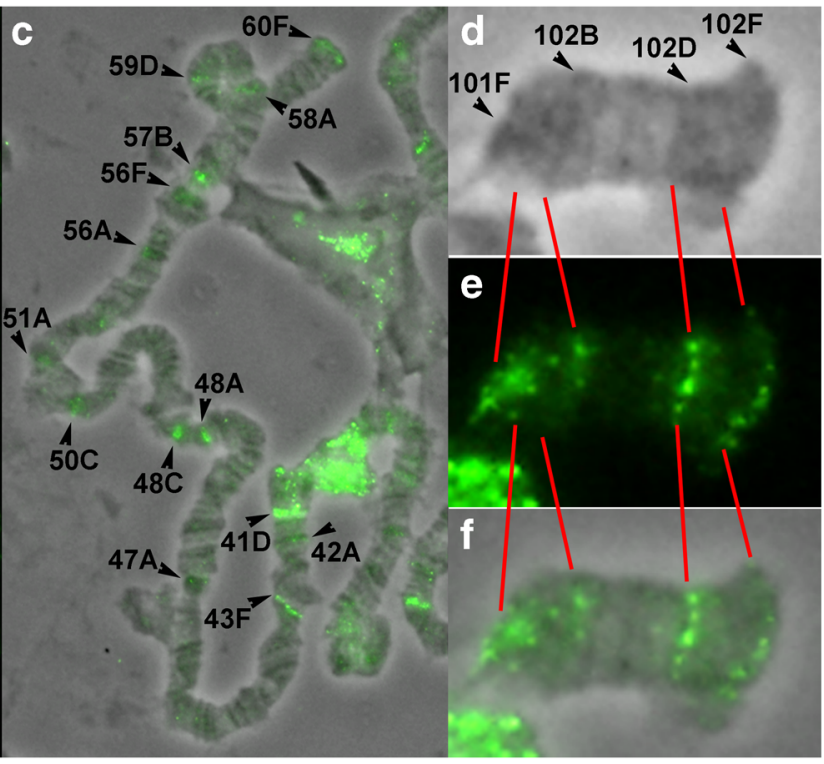

as control of proper SUUR localization in the dot chromosome. d Phase contrast of the fourth chromosome. e SUUR localization in the fourth chromosome $\mathbf{f}$ merged image of phase contrast and SUUR localization in the fourth chromosome 
a

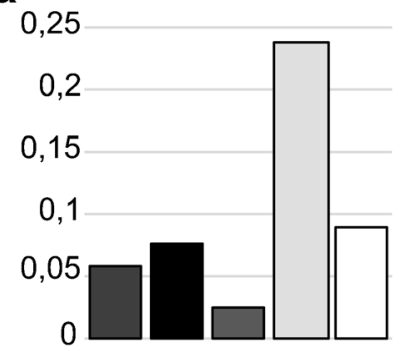

b

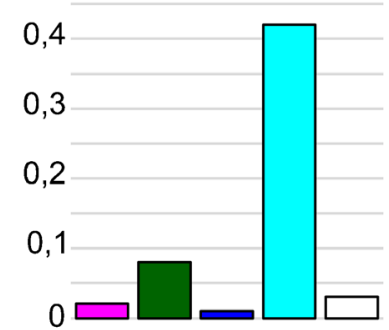

C

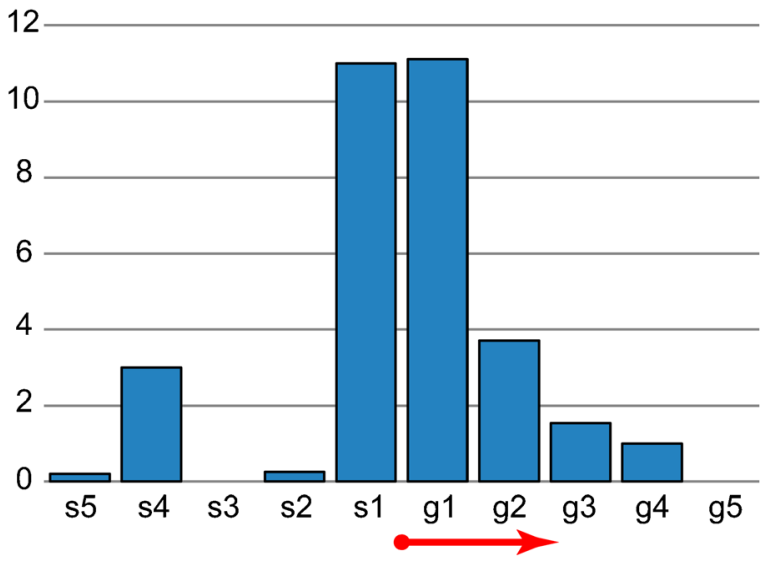

d

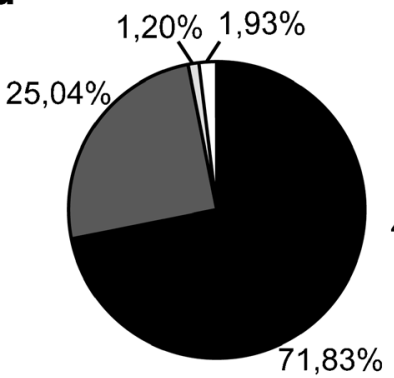

e

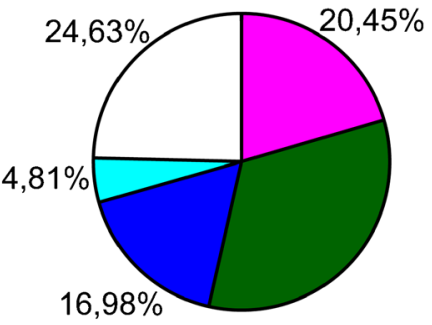

Fig. 13 Mobile element distribution in the fourth chromosome. $P$-element distribution: a in the cytological structures of the fourth chromosome, with the first column corresponding to all the bands of the fourth chromosome, and further - as shown in Fig. 2b; b in the four chromatin types of the fourth chromosome. The ordinate shows the density in $\mathrm{pcs} / \mathrm{kb}$. c $P$-element distribution relative to genes beginning in the interbands of the fourth chromosome. The interbands are divided into fragments of $200 \mathrm{bp}$ from the beginning of the gene towards the intergenic spacer (s1-s5) and towards the structural part of the gene (g1-g5); the genes are aligned with respect to the beginning. The graph shows five fragments in each direction. The ordinate shows the total number of sites in these fragments, normalized by the number of the gene transcripts. The red arrow shows the start and direction of the genes. The distribution of the 1360 element: $\mathbf{d}$ in the bands and interbands; $\mathbf{e}$ in the four chromatin types of the fourth chromosome.

We have shown that in the fourth chromosome the portion of dense ruby chromatin corresponding to black polygenic bands is $21 \%$ (Fig. 1a), which is about two times smaller than the ruby portion in the full genome of Drosophila (Zhimulev et al. 2014). The fraction of open aquamarine chromatin in the a

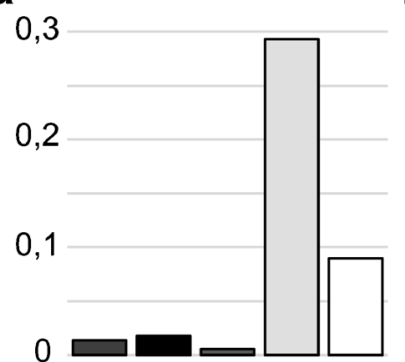

b

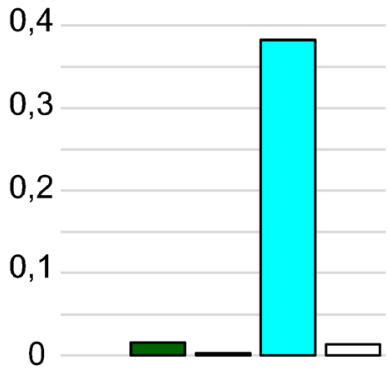

C

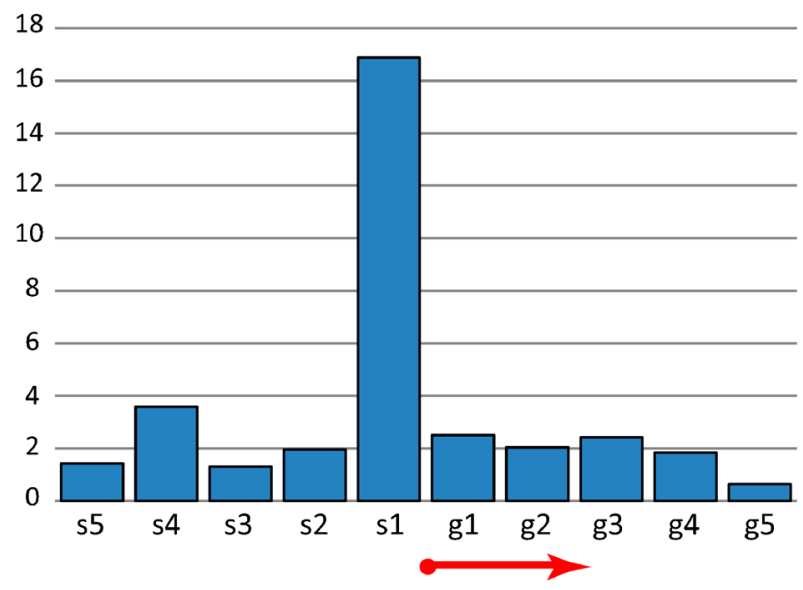

Fig. 14 Distribution of the DNaseI hypersensitivity sites (DHS): $\mathbf{a}$ in the cytological structures of the fourth chromosome, with the first column corresponding to all the bands of the fourth chromosome, and further - as shown in Fig. 2b; $\mathbf{b}$ in the four chromatin types of the fourth chromosome. The ordinate shows the density in $\mathrm{pcs} / \mathrm{kb}$. c DHS distribution (modENCODE data) in S2 cells relative to genes beginning in the interbands of the fourth chromosome. The interbands are divided into fragments of $200 \mathrm{bp}$ from the beginning of the gene towards the intergenic spacer (s1-s5) and towards the structural part of the gene (g1-g5); the genes are aligned with respect to the beginning. The graph shows five fragments in each direction. The ordinate shows the total number of sites in these fragments, normalized by the number of the gene transcripts. The red arrow shows the start and direction of the genes.

fourth chromosome is $12 \%$ (Fig. 1a), and it basically corresponds to interbands (Fig. 1c) and contains $5^{\prime}$ UTRs of housekeeping genes (Fig. 6a, Table S3), as in the whole genome. Coding parts of these genes are embedded in gray bands (Fig. 6a, Table S3), largely consisting of lazurite and malachite chromatin (Fig. 2d). These two types of chromatin at the genome level are supposed to be of intermediate compaction, since they are moderately enriched in certain molecular characteristics of both active and repressive chromatin (Boldyreva et al. 2017). In addition, malachite chromatin located at the borders of the intercalary heterochromatin bands forms a transition zone between these compact black bands and more loose areas (Khoroshko et al. 2016).

The gaps (areas where the model does not output certain chromatin type) in the fourth chromosome occupy more than $20 \%$ (Fig. 1a), whereas in the whole genome, these areas of uncertainty are only about 5\% (Boldyreva et al. 2017). 
Perhaps this is due to the fact that the protein distribution data has a smaller coverage for the fourth chromosome.

We showed that, according to the whole genome project data, four chromatin types of the fourth chromosome are enriched in marks of closed chromatin associated with gene repression, and depleted in many proteins and histone modifications of open chromatin relative to the rest of the genome (Table 1). Among other things, relative to the rest of the genome, aquamarine and lazurite chromatin of the fourth chromosome are enriched in the POF protein unique for this chromosome, and the heterochromatin protein HP1a (Table 1).

Previously, it has been shown that HP1a protein is localized not only in such typical heterochromatin regions as telomeres and pericentric heterochromatin, but also in some euchromatic loci and in the fourth chromosome of Drosophila, and that in the fourth polytene chromosome, this protein shows banded pattern (James et al. 1989). POF localization in the fourth chromosome has been shown to be approximately complementary to DAPI bands, i.e., it predominantly corresponds to interbands, and it is absent in the centromere of this chromosome (Larsson et al. 2001). Using chromatin immunoprecipitation, it has been shown that POF binds primarily to genes (Johansson et al. 2007a). In the same paper, it has been demonstrated that POF and HP1 are colocalized at the cytological level. A finer study using high-resolution ChIP experiment has revealed the distribution profiles of these two proteins. This study has shown that POF specifically binds to genes with a strong exon preference, and HP1a has a similar profile with a higher basal level of enrichment in the intergenic spacers and an additional peak in the promoter regions of occupied genes (Johansson et al. 2007b). The presence of the RNA-binding domain in the POF protein agrees with the RNA-seq data that indicate the cotranscriptional binding of this protein to the fourth chromosome nascent RNA and spliced RNA (Johansson et al. 2012). Improved polytene chromosome staining technique has revealed three additional POF binding sites: 2L:31 in in both males and females (Lundberg et al. 2013b), and PoX1 and PoX2 on the X chromosome in females (Lundberg et al. 2013a). Whole genome data on POF distribution in salivary gland polytene chromosomes obtained by ChIP-chip experiment demonstrate these three peaks (Lundberg et al. 2013a; Johansson and Larsson 2014). Recently, an optimal autonomous POF target has been reported to comprise a gene and a block of X chromosomelinked satellites 1.688 downstream this gene; thereby, the fourth chromosome seems to bear a cooperative effect of a large number of suboptimal combination of genes with repeats (Kim et al. 2018).

For a long time, HP1a has been considered as a heterochromatin protein (James and Elgin 1986). However, it plays an unusual role in the functioning of the fourth chromosome chromatin. On the one hand, this protein is necessary for the repression of transposons that are inserted in the fourth chromosome, and mutations in the $S u(v a r) 2-5$ gene lead to loss of silencing of the reporter gene in such construction (Sun et al. 2000; Riddle and Elgin 2006). On the other hand, it has been shown that HP1a binds to promoters and within the bodies of the active genes of the fourth chromosome (Johansson et al. 2007b; Figueiredo et al. 2012). HP1a is an indispensable factor for maintaining open chromatin state in promoter regions of the dot chromosome genes (Cryderman et al. 2011). Gene promoters usually located in repressive environment are held in open conformation if only they are packaged in HP1a-containing chromatin, and the transcription of such genes is not canceled by H3K9me2 (Cryderman et al. 2011). It has been shown that there are two mechanisms for HP1a attracting to the fourth chromosome: POFdependent recruiting of HP1a in the active gene areas, and POF-independent binding mechanism in the silent gene domains and clusters of repeats (Riddle et al. 2012). The specific high enrichment of actively transcribed gene coding parts in HP1a on the fourth chromosome positively regulates gene expression, promoting the transcription elongation and suppressing RNA polymerase pausing at the $5^{\prime}$ ends of genes (Riddle et al. 2012).

Linking of the cytological map of the D. melanogaster fourth polytene chromosome to the genomic coordinates enabled us to clarify the localization of HP1a and POF proteins with respect to the bands and interbands of the chromosome studied. Our data show that these proteins are mainly located in the interbands and gray bands of the dot chromosome (Figs. 8 and 9). According to the model of four chromatin states (Zhimulev et al. 2014; current investigation), HP1a and POF are most abundant in lazurite and aquamarine chromatin, which correspond to gray bands and interbands, respectively (Figs. 8 and 9). This result agrees with the previously published data that POF and HP1a are colocalized with the fourth chromosome genes, and the more active the transcription is, the stronger the binding is (Johansson et al. 2007b), since aquamarine chromatin corresponds to the promoters of ubiquitously active genes, and lazurite chromatin corresponds to the coding parts of these genes (Fig. 6a).

Recently, Kolesnikova and coworkers have determined the genomic coordinates for all black ruby bands of polytene chromosome $2 \mathrm{R}$ and have shown that the distribution of repressive chromatin marks H3K27me3 and SUUR is a good criterion for determining the boundaries of black bands (Kolesnikova et al. 2018). Using ChIP data on H3K27me3 localization in Drosophila larval salivary glands published earlier (Sher et al. 2012; GEO: GSE31897; Fig. S19i), we determined that in the fourth chromosome, only black bands containing large blocks of ruby chromatin are enriched with H3K27me3 (Fig. 11; Fig. S19c, d, i). The only exception is the first high peak of $\mathrm{H} 3 \mathrm{~K} 27 \mathrm{me} 3$ in the interval between black bands proximal to the centromere (Fig. S19c, d, i). As for 
SUUR protein, we faced with a contradiction between the DamID data published earlier (Filion et al. 2010; Maksimov et al. 2014; Posukh et al. 2017; Fig. S19j-p) and immunolocalization of this protein on polytene chromosome squashes (Fig. 12). The DamID data on salivary glands (Posukh et al. 2017) show SUUR binding nearly to the whole fourth polytene chromosome (Fig. S191), while immunostaining of polytene chromosome squashed preparations detects up to four discrete signals of this protein: near the centromere and telomere regions, and two bands in 102B and $102 \mathrm{D}$ regions (Fig. 12). We assume that such DamID results may be due to short-term interaction of SUUR with HP1a covering the fourth chromosome (Pindyurin et al. 2008). However, this issue is yet to be addressed.

$P$-elements (Fig. 13a-c), DNase I hypersensitivity sites (Fig. 14) and ORC2 protein of replication complex binding sites (Fig. 7) in the fourth chromosome are predominantly localized in open aquamarine chromatin of the interbands. These genome elements demonstrate a distribution peak around the 5'UTRs of genes localized in aquamarine chromatin. This data correlate with the whole genome data (Zhimulev et al. 2014). In contrast, the 1360 element, characteristic of the fourth chromosome, tends to occupy band chromatin types: ruby, lazurite, and malachite (Fig. 13d, e).

Thus, the fourth chromosome of $D$. melanogaster differs from the rest of the genome in a large number of gray bands, and the absence of large black intercalary heterochromatin bands. Another difference is the presence of special epigenetic mechanisms of the gene expression regulation by POF, HP1a, and dSETDB1 proteins. At the same time, the results obtained show that in general, the band organization of the fourth chromosome coincides with the organization of the rest of the genome.

Acknowledgements We thank Elena S. Belyaeva for the fly stock granted and Tatyana D. Kolesnikova for SUUR immunolocalization polytene chromosome preparations.

Funding The work was supported by the fundamental scientific research program on the project \#0310-2018-0010 in fluorescent in situ hybridization, the Russian Science Foundation project \#14-14-00934 for works on comparison of cytological and physical maps of the Drosophila fourth chromosome, and the Russian Foundation for Basic Research project \#17-00-00284 regarding software development and definition of transposable element localization in cytological structures of the dot chromosome.

\section{Compliance with ethical standards}

Conflict of interest The authors declare that they have no conflict of interest.

Open Access This article is distributed under the terms of the Creative Commons Attribution 4.0 International License (http:// creativecommons.org/licenses/by/4.0/), which permits unrestricted use, distribution, and reproduction in any medium, provided you give appropriate credit to the original author(s) and the source, provide a link to the Creative Commons license, and indicate if changes were made.

\section{References}

Andreyeva EN, Kolesnikova TD, Belyaeva ES, Glaser RL, Zhimulev IF (2008) Local DNA underreplication correlates with accumulation of phosphorylated $\mathrm{H} 2 \mathrm{Av}$ in the Drosophila melanogaster polytene chromosomes. Chromosom Res 16:851-862. https://doi.org/10.1007/s10577008-1244-4

Andreyeva EN, Kolesnikova TD, Demakova OV, Mendez-Lago M, Pokholkova GV, Belyaeva ES, Rossi F, Dimitri P, Villasante A, Zhimulev IF (2007) High-resolution analysis of Drosophila heterochromatin organization using SuUR Su(var)3-9 double mutants. Proc Natl Acad Sci U S A 104:12819-12824. https://doi.org/10. 1073/pnas.0704690104

Ashburner M, Golic KG, Hawley RS (2005) Drosophila: a laboratory handbook, 2nd edn. Cold Spring Harbor Laboratory Press, Cold Spring Harbor

Boldyreva LV, Goncharov FP, Demakova OV, Zykova TY, Levitsky VG, Kolesnikov NN, Pindyurin AV, Semeshin VF, Zhimulev IF (2017) Protein and genetic composition of four chromatin types in Drosophila melanogaster cell lines. Curr Genomics 18:214-226. https://doi.org/10.2174/ 1389202917666160512164913

Bridges CB (1935a) Salivary chromosome maps with a key to the banding of the chromosomes of Drosophila melanogaster. J Hered 26:60-64

Bridges CB (1935b) Cytological data on chromosome four of Drosophila melanogaster. Trans Dyn Dev 10:463-473

Brower-Toland B, Riddle NC, Jiang H, Huisinga KL, Elgin SC (2009) Multiple SET methyltransferases are required to maintain normal heterochromatin domains in the genome of Drosophila melanogaster. Genetics 181:1303-1319. https://doi.org/10.1534/ genetics.108.100271

Chintapalli VR, Wang J, Dow JAT (2007) Using FlyAtlas to identify better Drosophila melanogaster models of human disease. Nat Genet 39(6):715-720

Corona DF, Siriaco G, Armstrong JA, Snarskaya N, McClymont SA, Scott MP, Tamkun JW (2007) ISWI regulates higher-order chromatin structure and histone H1 assembly in vivo. PLoS Biol 5:e232. https://doi.org/10.1371/journal.pbio.0050232

Cryderman DE, Vitalini MW, Wallrath LL (2011) Heterochromatin protein $1 \mathrm{a}$ is required for an open chromatin structure. Transcription 2: 95-99. https://doi.org/10.4161/trns.2.2.14687

Czermin B, Melfi R, McCabe D, Seitz V, Imhof A, Pirrotta V (2002) Drosophila enhancer of Zeste/ESC complexes have a histone H3 methyltransferase activity that marks chromosomal Polycomb sites. Cell 111(2):185-196

Demakov SA, Semeshin VF, Zhimulev IF (1993) Cloning and molecular genetic analysis of Drosophila melanogaster interband DNA. Mol Gen Genet 238:437-443

Demakov SA, Vatolina TY, Babenko VN, Semeshin VF, Belyaeva ES, Zhimulev IF (2011) Protein composition of interband regions in polytene and cell line chromosomes of Drosophila melanogaster. BMC Genomics 12:566. https://doi.org/10.1186/1471-2164-12-566

Demakova OV, Pokholkova GV, Kolesnikova TD, Demakov SA, Andreyeva EN, Belyaeva ES, Zhimulev IF (2007) The SU(VAR)3-9/HP1 complex differentially regulates the compaction state and degree of underreplication of $\mathrm{X}$ chromosome pericentric 
heterochromatin in Drosophila melanogaster. Genetics 175:609620. https://doi.org/10.1534/genetics.106.062133

Deuring R, Fanti L, Armstrong JA, Sarte M, Papoulas O, Prestel M, Daubresse G, Verardo M, Moseley SL, Berloco M, Tsukiyama T, Wu C, Pimpinelli S, Tamkun JW (2000) The ISWI chromatinremodeling protein is required for gene expression and the maintenance of higher order chromatin structure in vivo. Mol Cell 5:355365. https://doi.org/10.1016/S1097-2765(00)80430-X

Eaton ML, Prinz JA, MacAlpine HK, Tretyakov G, Kharchenko PV, MacAlpine DM (2011) Chromatin signatures of the Drosophila replication program. Genome Res 21(2):164-174. https://doi.org/10. 1101/gr.116038.110

Elgin SC, Reuter G (2013) Position-effect variegation, heterochromatin formation, and gene silencing in Drosophila. Cold Spring Harb Perspect Biol 5(8):a017780. https://doi.org/10.1101/cshperspect. a017780

Figueiredo ML, Philip P, Stenberg P, Larsson J (2012) HP1a recruitment to promoters is independent of $\mathrm{H} 3 \mathrm{~K} 9$ methylation in Drosophila melanogaster. PLoS Genet 8:e1003061. https://doi.org/10.1371/ journal.pgen.1003061

Filion GJ, van Bemmel JG, Braunschweig U, Talhout W, Kind J, Ward LD, Brugman W, de Castro IJ, Kerkhoven RM, Bussemaker HJ, van Steensel B (2010) Systematic protein location mapping reveals five principal chromatin types in Drosophila cells. Cell 143:212-224. https://doi.org/10.1016/j.cell.2010.09.009

Gatti M, Pimpinelli S, Santini G (1976) Characterization of Drosophila heterochromatin. I. Staining and decondensation with Hoechst 33258 and Quinacrine. Chromosoma 57:351-375

Gortchakov AA, Eggert H, Gan M, Mattow J, Zhimulev IF, Saumweber $\mathrm{H}$ (2005) Chriz, a chromodomain protein specific for the interbands of Drosophila melanogaster polytene chromosomes. Chromosoma 114(1):54-66. https://doi.org/10.1007/s00412-005-0339-3

Hou C, Li L, Qin ZS, Corces VG (2012) Gene density, transcription, and insulators contribute to the partition of the Drosophila genome into physical domains. Mol Cell 48:471-484. https://doi.org/10.1016/j. molcel.2012.08.031

James TC, Eissenberg JC, Craig C, Dietrich V, Hobson A, Elgin SC (1989) Distribution patterns of HP1, a heterochromatin-associated nonhistone chromosomal protein of Drosophila. Eur J Cell Biol 50: $170-180$

James TC, Elgin SC (1986) Identification of a nonhistone chromosomal protein associated with heterochromatin in Drosophila melanogaster and its gene. Mol Cell Biol 6:3862-3872

Johansson AM, Larsson J (2014) Genome-wide mapping of Painting of fourth on Drosophila melanogaster salivary gland polytene chromosomes. Genom Data 2:63-65. https://doi.org/10.1016/j.gdata.2014. 04.007

Johansson AM, Stenberg P, Allgardsson A, Larsson J (2012) POF regulates the expression of genes on the fourth chromosome in Drosophila melanogaster by binding to nascent RNA. Mol Cell Biol 32:2121-2134. https://doi.org/10.1128/MCB.06622-11

Johansson AM, Stenberg P, Bernhardsson C, Larsson J (2007a) Painting of fourth and chromosome-wide regulation of the 4th chromosome in Drosophila melanogaster. EMBO J 26:2307-2316. https://doi. org/10.1038/sj.emboj.7601604

Johansson AM, Stenberg P, Pettersson F, Larsson J (2007b) POF and HP1 bind expressed exons, suggesting a balancing mechanism for gene regulation. PLoS Genet 3:e209. https://doi.org/10.1371/journal. pgen.0030209

Kharchenko PV, Alekseyenko AA, Schwartz YB, Minoda A, Riddle NC, Ernst J, Sabo PJ, Larschan E, Gorchakov AA, Gu T, Linder-Basso D, Plachetka A, Shanower G, Tolstorukov MY, Luquette LJ, Xi R, Jung YL, Park RW, Bishop EP, Canfield TK, Sandstrom R, Thurman RE, MacAlpine DM, Stamatoyannopoulos JA, Kellis M, Elgin SCR, Kuroda MI, Pirrotta V, Karpen GH, Park PJ (2011) Comprehensive analysis of the chromatin landscape in Drosophila melanogaster. Nature 471:480-485. https://doi.org/10.1038/ nature09725

Kholodilov NG, Bolshakov VN, Blinov VM, Solovyov VV, Zhimulev IF (1988) Intercalary heterochromatin in Drosophila. III. Homology between DNA sequences from the Y chromosome, bases of polytene chromosome limbs, and chromosome 4 of $D$. melanogaster. Chromosoma 97:247-253

Khoroshko VA, Levitsky VG, Zykova TY, Antonenko OV, Belyaeva ES, Zhimulev IF (2016) Chromatin heterogeneity and distribution of regulatory elements in the late-replicating intercalary heterochromatin domains of Drosophila melanogaster chromosomes. PLoS One 11:e0157147. https://doi.org/10.1371/journal.pone.0157147

Khoroshko VA, Zykova TY, Popova OO, Zhimulev IF (2018) Border structure of intercalary heterochromatin bands of Drosophila melanogaster polytene chromosomes. Dokl Biochem Biophys 479:114-117. https://doi.org/10.1134/S1607672918020163

Kim M, Ekhteraei-Tousi S, Lewerentz J, Larsson J (2018) The X-linked 1.688 satellite in Drosophila melanogaster promotes specific targeting by Painting of fourth. Genetics 208:623-632. https://doi. org/10.1534/genetics.117.300581

King RC (1975) Drosophila melanogaster: an introduction. In: King RC (ed) Handbook of genetics, Invertebrates of genetic interest, vol 3. Plenum Press, New York, pp 625-652

Kolesnikova TD (2018) Banding pattern of polytene chromosomes as a representation of universal principles of chromatin organization into topological domains. Biochem Mosc 83(4):338-349

Kolesnikova TD, Goncharov FP, Zhimulev IF (2018) Similarity in replication timing between polytene and diploid cells is associated with the organization of the Drosophila genome. PLoS One 13: e0195207. https://doi.org/10.1371/journal.pone.0195207

Kolesnikova TD, Posukh OV, Andreyeva EN, Bebyakina DS, Ivankin AV, Zhimulev IF (2013) Drosophila SUUR protein associates with PCNA and binds chromatin in a cell cycle-dependent manner. Chromosoma 122:55-66. https://doi.org/10.1007/s00412-0120390-9

Kurenova EV, Leìbovich BA, Bass IA, Bebikhov DV, Pavlova MN, Danilevskaia ON (1990) Hoppel-family of mobile elements of Drosophila melanogaster, flanked by short inverted repeats and having preferential localization in the heterochromatin regions of the genome. Genetika 26(10):1701-1712

Larsson J, Chen JD, Rasheva V, Rasmuson-Lestander A, Pirrotta V (2001) Painting of fourth, a chromosome-specific protein in Drosophila. Proc Natl Acad Sci U S A 98:6273-6278. https://doi. org/10.1073/pnas.111581298

Lawrence M, Gentleman R, Carey V (2009) rtracklayer: an R package for interfacing with genome browsers. Bioinformatics 25:1841-1842. https://oi.org/10.1093/bioinformatics/btp328

Lawrence M, Huber W, Pagès H, Aboyoun P, Carlson M, Gentleman R, Morgan MT, Carey VJ (2013) Software for computing and annotating genomic ranges. PLoS Comput Biol 9:e1003118. https://doi.org/ 10.1371/journal.pcbi.1003118

Locke J, Howard LT, Aippersbach N, Podemski L, Hodgetts RB (1999) The characterization of DINE-1, a short, interspersed repetitive element present on chromosome and in the centric heterochromatin of Drosophila melanogaster. Chromosoma 108:356-366

Locke J, McDermid HE (1993) Analysis of Drosophila chromosome 4 using pulsed field gel electrophoresis. Chromosoma 102:718-723

Lundberg LE, Kim M, Johansson AM, Faucillion ML, Josupeit R, Larsson J (2013a) Targeting of Painting of fourth to roX1 and roX2 proximal sites suggests evolutionary links between dosage compensation and the regulation of the fourth chromosome in Drosophila melanogaster. G3 (Bethesda) 3:1325-1334. https://doi. org $/ 10.1534 / \mathrm{g} 3.113 .006866$

Lundberg LE, Stenberg P, Larsson J (2013b) HP1a, Su(var)3-9, SETDB1 and POF stimulate or repress gene expression depending on genomic position, gene length and expression pattern in Drosophila 
melanogaster. Nucleic Acids Res 41:4481-4494. https://doi.org/10. 1093/nar/gkt158

Madigan JP, Chotkowski HL, Glaser RL (2002) DNA double-strand break-induced phosphorylation of Drosophila histone variant $\mathrm{H} 2 \mathrm{Av}$ helps prevent radiation-induced apoptosis. Nucleic Acids Res 30:3698-3705

Maksimov DA, Koryakov DE, Belyakin SN (2014) Developmental variation of the SUUR protein binding correlates with gene regulation and specific chromatin types in D. melanogaster. Chromosoma 123: 253-264. https://doi.org/10.1007/s00412-013-0445-6

Mehrotra S, McKim KS (2006) Temporal analysis of meiotic DNA double-strand break formation and repair in Drosophila females. PLoS Genet 2(11):e200. https://doi.org/10.1371/journal.pgen. 0020200

Milon B, Sun Y, Chang W, Creasy T, Mahurkar A, Shetty A, Nurminsky D, Nurminskaya M (2014) Map of open and closed chromatin domains in Drosophila genome. BMC Genomics 15:988. https://doi. org/10.1186/1471-2164-15-988

Morgan TH, Bridges CB, Schultz J (1934) Constitution of the germinal material in relation to heredity. Yearbook Carnegie Inst Wash 33: 274-280

Pimpinelli S, Santini G, Gatti M (1976) Characterization of Drosophila heterochromatin. II. C- and N-banding. Chromosoma 57:377-386

Pindyurin AV, Boldyreva LV, Shloma VV, Kolesnikova TD, Pokholkova GV, Andreyeva EN, Kozhevnikova EN, Ivanoschuk IG, Zarutskaya EA, Demakov SA, Gorchakov AA, Belyaeva ES, Zhimulev IF (2008) Interaction between the Drosophila heterochromatin proteins SUUR and HP1. J Cell Sci 121:1693-1703. https://doi.org/10.1242/ jes.018655

Pokholkova GV, Makunin IV, Belyaeva ES, Zhimulev IF (1993) Obervations on the induction of position effect variegation of euchromatic genes in Drosophila melanogaster. Genetics 134(1):231242

Posukh OV, Maksimov DA, Laktionov PP, Koryakov DE, Belyakin SN (2017) Functional dissection of Drosophila melanogaster SUUR protein influence on $\mathrm{H} 3 \mathrm{~K} 27 \mathrm{me} 3$ profile. Epigenetics Chromatin 10:56. https://doi.org/10.1186/s13072-017-0163-z

R Core Team (2017) R: a language and environment for statistical computing. https://www.R-project.org/

Reiss D, Quesneville H, Nouaud D, Andrieu O, Anxolabehere D (2003) Hoppel, a $P$-like element without introns: a $P$-element ancestral structure or a retrotranscription derivative? Mol Biol Evol 20(6): 869-879. https://doi.org/10.1093/molbev/msg090

Riddle NC, Elgin SC (2006) The dot chromosome of Drosophila: insights into chromatin states and their change over evolutionary time. Chromosom Res 14:405-416. https://doi.org/10.1007/s10577-0061061-6

Riddle NC, Elgin SC (2018) The Drosophila dot chromosome: where genes flourish amidst repeats. Genetics 210(3):757-772. https://doi. org/10.1534/genetics.118.301146

Riddle NC, Jung YL, Gu T, Alekseyenko AA, Asker D, Gui H, Kharchenko PV, Minoda A, Plachetka A, Schwartz YB, Tolstorukov MY, Kuroda MI, Pirrotta V, Karpen GH, Park PJ, Elgin SCR (2012) Enrichment of HP1a on Drosophila chromosome 4 genes creates an alternate chromatin structure critical for regulation in this heterochromatic domain. PLoS Genet 8:e1002954. https://doi.org/10.1371/journal.pgen.1002954

Riddle NC, Leung W, Haynes KA, Granok H, Wuller J, Elgin SCR (2008) An investigation of heterochromatin domains on the fourth chromosome of Drosophila melanogaster. Genetics 178:11771191. https://doi.org/10.1534/genetics.107.081828

Riddle NC, Shaffer CD, Elgin SC (2009) A lot about a little dot - lessons learned from Drosophila melanogaster chromosome 4. Biochem Cell Biol 87:229-241. https://doi.org/10.1139/O08-119
Sandler L, Szauter P (1978) The effect of recombination-defective meiotic mutants on fourth-chromosome crossing over in Drosophila melanogaster. Genetics 90:699-712

Saura AO, Cuenca JB, Heino TI, de Frutos R, Sorsa V (2002) The polytene dot chromosome of Drosophila: D. melanogaster and D. subobscura. Chromosoma 111:273-283. https://doi.org/10. 1007/s00412-002-0209-1

Semeshin VF, Belyaeva ES, Shloma VV, Zhimulev IF (2004) Electron microscopy of polytene chromosomes. Methods Mol Biol 247:305324

Semeshin VF, Belyaeva ES, Zhimulev IF, Lis JT, Richards G, Bourouis M (1986) Electron microscopical analysis of Drosophila polytene chromosomes. IV. Mapping of morphological structures appearing as a result of transformation of DNA sequences into chromosomes. Chromosoma 93:461-468

Semeshin VF, Demakov SA, Perez Alonso M, Belyaeva ES, Bonner JJ, Zhimulev IF (1989) Electron microscopical analysis of Drosophila polytene chromosomes. V. Characteristics of structures formed by transposed DNA segments of mobile elements. Chromosoma 97: 396-412

Seum C, Reo E, Peng H, Rauscher FJ, Spierer P, Bontron S (2007) Drosophila SETDB1 is required for chromosome 4 silencing. PLoS Genet 3:e76. https://doi.org/10.1371/journal.pgen.0030076

Sher N, Bell GW, Li S, Nordman J, Eng T, Eaton ML, MacAlpine DM, Orr-Weaver TL (2012) Developmental control of gene copy number by repression of replication initiation and fork progression. Genome Res 22:64-75. https://doi.org/10.1101/gr.126003.111

Slawson EE, Shaffer CD, Malone CD, Leung W, Kellmann E, Shevchek RB, Craig CA, Bloom SM, Bogenpohl J, Dee J, Morimoto ETA, Myoung J, Nett AS, Ozsolak F, Tittiger ME, Zeug A, Pardue ML, Buhler J, Mardis ER, Elgin SCR (2006) Comparison of dot chromosome sequences from $D$. melanogaster and $D$. virilis reveals an enrichment of DNA transposon sequences in heterochromatic domains. Genome Biol 7:R15. https://doi.org/10.1186/gb-2006-7-2r15

Slizynski BM (1944) A revised map of salivary gland chromosome 4 of Drosophila melanogaster. J Hered 35:322-325

Stadler MR, Haines JE, Eisen MB (2017) Convergence of topological domain boundaries, insulators, and polytene interbands revealed by high-resolution mapping of chromatin contacts in the early Drosophila melanogaster embryo. Elife 6:e29550

Sun FL, Cuaycong MH, Craig CA, Wallrath LL, Locke J, Elgin SC (2000) The fourth chromosome of Drosophila melanogaster: interspersed euchromatic and heterochromatic domains. Proc Natl Acad Sci U S A 97:5340-5345. https://doi.org/10.1073/pnas.090530797

The modENCODE Consortium, Roy S, Ernst J et al (2010) Identification of functional elements and regulatory circuits by Drosophila modENCODE. Science 330:1787-1797. https://doi.org/10.1126/ science. 1198374

Tzeng TY, Lee CH, Chan LW, Shen CK (2007) Epigenetic regulation of the Drosophila chromosome 4 by the histone H3K9 methyltransferase dSETDB1. Proc Natl Acad Sci U S A 104:12691-12696. https:// doi.org/10.1073/pnas.0705534104

Vatolina TY, Boldyreva LV, Demakova OV, Demakov SA, Kokoza EB, Semeshin VF, Babenko VN, Goncharov FP, Belyaeva ES, Zhimulev IF (2011a) Identical functional organization of nonpolytene and polytene chromosomes in Drosophila melanogaster. PLoS One 6: e25960. https://doi.org/10.1371/journal.pone.0025960

Vatolina TI, Demakov SA, Semeshin VF, Makunin IV, Babenko VN, Beliaeva ES, Zhimulev IF (2011b) Identification and molecular genetic characterization of the polytene chromosome interbands in Drosophila melanogaster. Genetika 47:597-609

Zhimulev IF, Belyaeva ES, Makunin IV, Pirrotta V, Volkova E, Alekseyenko A, Andreyeva E, Makarevich G, Boldyreva L, Nanayev R, Demakova O (2003) Influence of the SuUR gene on intercalary heterochromatin in Drosophila melanogaster polytene 
chromosomes. Chromosoma 111:377-398. https://doi.org/10.1007/ s00412-002-0218-0

Zhimulev IF, Boldyreva LV, Demakova OV, Poholkova GV, Khoroshko VA, Zykova TY, Lavrov SA, Belyaeva ES (2016) Drosophila polytene chromosome bands formed by gene introns. Dokl Biochem Biophys 466:57-60. https://doi.org/10.1134/S1607672916010178

Zhimulev IF, Zykova TY, Goncharov FP, Khoroshko VA, Demakova OV, Semeshin VF, Pokholkova GV, Boldyreva LV, Demidova DS, Babenko VN, Demakov SA, Belyaeva ES (2014) Genetic organization of interphase chromosome bands and interbands in Drosophila melanogaster. PLoS One 9:e101631. https://doi.org/10.1371/ journal.pone. 0101631

Zykova TY, Levitsky VG, Belyaeva ES, Zhimulev IF (2018) Polytene chromosomes - a portrait of functional organization of the Drosophila genome. Curr Genomics 19:179-191. https://doi.org/ $10.2174 / 1389202918666171016123830$

Publisher's note Springer Nature remains neutral with regard to jurisdictional claims in published maps and institutional affiliations. 